\title{
Sensitivity Analysis of Grain Surface Chemistry to Binding Energies of Ice Species
}

\author{
E. M. Penteado ${ }^{1}$, C. Walsh ${ }^{2,3}$, and H. M. Cuppen ${ }^{1}$ \\ ${ }^{1}$ Radboud University, Institute for Molecules and Materials, Heyendaalseweg 135, NL-6525 AJ Nijmegen, The Netherlands; h.cuppen@science.ru.nl \\ ${ }^{2}$ School of Physics and Astronomy, University of Leeds, Leeds LS2 9JT, UK \\ ${ }^{3}$ Leiden Observatory, Leiden University P.O. Box 9513, 2300 RA Leiden, The Netherlands \\ Received 2016 October 20; revised 2017 May 9; accepted 2017 May 23; published 2017 July 24
}

\begin{abstract}
Advanced telescopes, such as ALMA and the James Webb Space Telescope, are likely to show that the chemical universe may be even more complex than currently observed, requiring astrochemical modelers to improve their models to account for the impact of new data. However, essential input information for gas-grain models, such as binding energies of molecules to the surface, have been derived experimentally only for a handful of species, leaving hundreds of species with highly uncertain estimates. We present in this paper a systematic study of the effect of uncertainties in the binding energies on an astrochemical two-phase model of a dark molecular cloud, using the rate equations approach. A list of recommended binding energy values based on a literature search of published data is presented. Thousands of simulations of dark cloud models were run, and in each simulation a value for the binding energy of hundreds of species was randomly chosen from a normal distribution. Our results show that the binding energy of $\mathrm{H}_{2}$ is critical for the surface chemistry. For high binding energies, $\mathrm{H}_{2}$ freezes out on the grain forming an $\mathrm{H}_{2}$ ice. This is not physically realistic, and we suggest a change in the rate equations. The abundance ranges found are in reasonable agreement with astronomical ice observations. Pearson correlation coefficients revealed that the binding energy of $\mathrm{HCO}, \mathrm{HNO}, \mathrm{CH}_{2}$, and $\mathrm{C}$ correlate most strongly with the abundance of dominant ice species. Finally, the formation route of complex organic molecules was found to be sensitive to the branching ratios of $\mathrm{H}_{2} \mathrm{CO}$ hydrogenation.
\end{abstract}

Key words: astrochemistry - galaxies: star formation - ISM: molecules

Supporting material: machine-readable table

\section{Introduction}

The new generation of telescopes, e.g., ALMA and the James Webb Space Telescope, will provide us with an unprecedented wealth of molecular information. For ALMA, this is at very high spatial resolution, which depends on both the observing frequency and the maximum baseline. Despite the fact that ALMA observes these molecules in the gas phase through rotational emission, many of the more complex saturated organic molecules are thought to originate from the mantles of dust grains, since either they or their precursors are formed through grain-surface chemistry.

To interpret all this information, it becomes more and more pressing to have reliable gas-grain chemical models. Astrochemical models have been developed over the decades to understand the molecular processes in the interstellar medium (ISM). Although different approaches have been applied, most studies use the rate equation method because of its simplicity and its advantageous computational time. The gas-phase part of these models has been extensively reviewed, and the error propagation of the input parameters in the form of rate constants for gas-phase reactions has been considered in the calculation of final abundances (Wakelam et al. 2010).

Here, we extend this effort to the grain-surface part of gas - grain codes by looking at the effect of uncertainties on the binding energy $\left(E_{\text {bind }}\right)$ of species to the grain, by applying the same methodology used for the gas-phase reaction rates as Wakelam et al. (2006, 2010). This parameter determines the upper value of the temperature range at which species are available for reactions on the grain surface. Moreover, since in most models the diffusion barrier is obtained from the binding energy, it also, indirectly, determines the onset temperature for surface reactions through the diffusive Langmuir-Hinshelwood mechanism.

Binding energies can be determined experimentally using the Temperature Programmed Desorption (TPD) technique, which will be briefly explained in the next section. But it is not straightforward to provide binding energy information for radical species using TPD, since these species are very short lived under laboratory conditions. In some cases, computational studies can provide valuable information. However, the first gas-grain chemical models (Tielens \& Hagen 1982; Hasegawa \& Herbst 1993b; Charnley 1997) predate TPD experiments. The binding energies in these early works were estimated from the polarizability of the molecule or atom, which provides an estimate of the strength of the van der Waals interaction with the bare grain surface.

In the present paper, we present an extensive literature search in which we arrive at recommended values for the binding energies and their uncertainties. Next, thousands of simulations are performed, where each simulation uses a set of binding energies that is randomly picked from a Gaussian distribution that considers the recommended binding energies and their uncertainties. The species that provide the strongest effect on the abundance of key ice components, such as $\mathrm{H}_{2} \mathrm{O}$, $\mathrm{CO}$ and $\mathrm{CH}_{3} \mathrm{OH}$, will be discussed and compared against ice observations.

\section{Binding Energies}

The chemical network used in the present work (Garrod et al. 2008; Drozdovskaya et al. 2014, 2015; Walsh et al. 2015, and references therein) contains 190 surface species for which we need to provide a binding energy. As mentioned before, 
Table 1

Experimentally Determined $E_{\text {bind }}$ Values and Specifics of Each Experiment

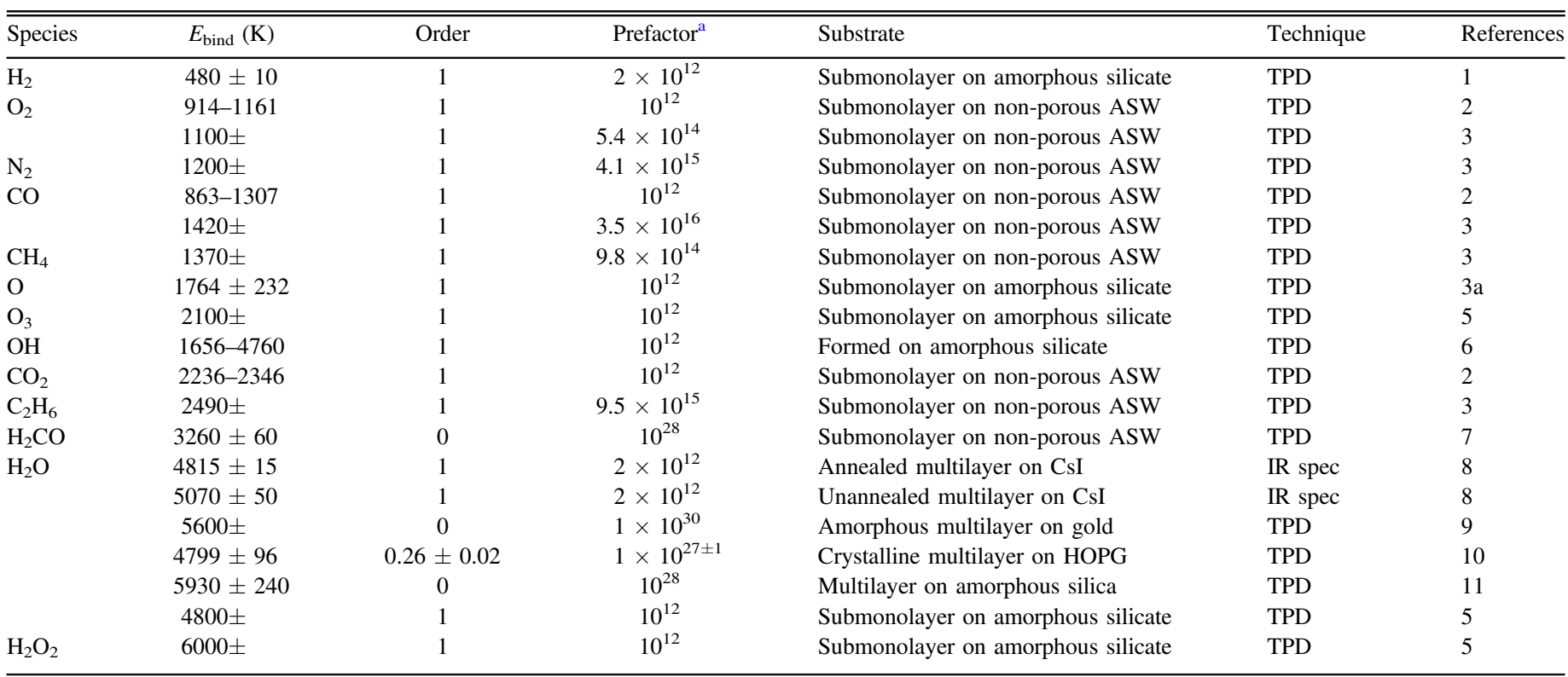

Note.

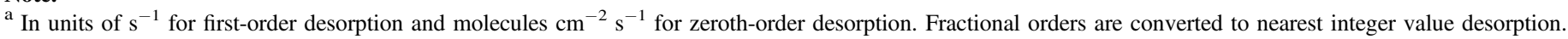

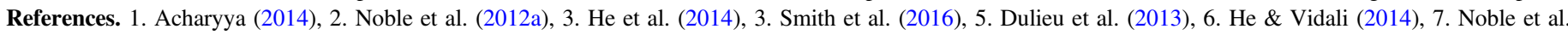
(2012b), 8. Sandford \& Allamandola (1988), 9. Fraser et al. (2001), 10. Brown \& Bolina (2007), 11. Collings et al. (2015).

binding energies are typically obtained by experiments that use the TPD technique. This well-established technique consists of two stages: first, the temperature of the substrate is kept constant while the species of interest is deposited; and second, the temperature is linearly increased until the species are desorbed from the substrate while the desorbing species are recorded using a mass spectrometer. Next, the Polanyi-Wigner equation

$$
\frac{d N}{d t}=-k_{m} N^{m} \exp \left[-\frac{E_{\mathrm{bind}, i}}{k_{\mathrm{B}} T}\right],
$$

where $N$ and $T$ are, respectively, the total number of absorbed species and the surface temperature, both at a certain time $t$, is fitted to the measured desorption spectra of a species $i$ to obtain $E_{\text {bind }, i}$, a prefactor, and in some cases also the desorption order $m$. Since the prefactor and binding energy are highly correlated, several experiments need to be performed with different amounts of predeposited species. The final results depend on the nature of the substrate from which the species desorbs. As an example, we would like to mention the reported experimental values of $E_{\text {bind }}$ for water, which may range from $4800 \mathrm{~K}$, as reported by Dulieu et al. (2013) from studies of formation of water on amorphous silicate surfaces, to $5930 \pm 240 \mathrm{~K}$, as reported by Collings et al. (2015), based on experiments of desorption from amorphous silica. Whether experiments are performed in the monolayer or multilayer regime and whether the deposited ice is pure, mixed, or layered also has an effect. In the multilayer regime, desorption occurs from the species itself and the effect of the underlying substrate becomes negligible as pointed out by Green et al. (2009). Most experiments concentrate their attention on a few important species, such as $\mathrm{H}_{2} \mathrm{O}, \mathrm{CO}, \mathrm{CO}_{2}$, and $\mathrm{CH}_{3} \mathrm{OH}$. Data on more complex species is far more sparse. Our collected data are presented in Table 1. Here, we take the binding energies from experiments with an amorphous water ice substrate where possible, as an attempt to use homogeneous data, since the differences between different substrates can be large, although smaller in the multilayer regime (Green et al. 2009). The quoted uncertainties in the table are a combination of experimental errors, fitting errors, and the intrinsic variety of the binding energies due to the inhomogeneity of the substrate. The latter is especially important in the case of amorphous substrates.

In some cases, computational studies can help derive binding energies. For instance, Al-Halabi \& van Dishoeck (2007) simulated adsorption of $\mathrm{H}$ atoms to amorphous solid water using classical trajectory calculations. The off-lattice kinetic Monte Carlo approach was used by Karssemeijer \& Cuppen (2014) to estimate the binding energies of $\mathrm{CO}$ and $\mathrm{CO}_{2}$. For the majority of species, however, there are still neither laboratory data nor computational estimates available.

In a few experimental studies, TPD spectra are presented in figures, but no binding energy data are provided by the authors. An example is the paper by Collings et al. (2004). They present an extensive experimental study of a collection of astrophysically relevant molecular species using the TPD technique. Three kinds of experiments were performed, differentiated according to the substrate used: (1) deposition of each species on a pure gold substrate, (2) deposition on a $\mathrm{H}_{2} \mathrm{O}$ substrate, and (3) co-deposition of each species with water forming a mixture as a substrate. Collings et al. (2004) classified the studied species into three categories based on their desorption behavior. One of these categories is the defined CO-like species, composed of $\mathrm{N}_{2}, \mathrm{O}_{2}$, and $\mathrm{CH}_{4}$. These very volatile species desorb similarly to $\mathrm{CO}$, presenting two desorption peaks. This is especially evident for $\mathrm{N}_{2}$ and $\mathrm{O}_{2}$. Molecules in the second 
Table 2

Estimated $T_{\text {des }}$ and $E_{\text {bind }}$ from TPD Experiments

\begin{tabular}{lcc}
\hline \hline Species & $T_{\text {des }}(\mathrm{K})$ & $E_{\text {bind }}(\mathrm{K})$ \\
\hline $\mathrm{O}_{2}$ & 29 & $865 \pm 55^{\mathrm{a}}$ \\
& 45 & $1342 \pm 65^{\mathrm{b}}$ \\
$\mathrm{N}_{2}$ & 30 & $895 \pm 55^{\mathrm{a}}$ \\
& 46 & $1305 \pm 70^{\mathrm{b}}$ \\
$\mathrm{C}_{2} \mathrm{H}_{2}$ & 70 & $2090 \pm 85$ \\
$\mathrm{H}_{2} \mathrm{~S}$ & 77 & $2296 \pm 90$ \\
$\mathrm{CO}_{2}$ & 78 & $2325 \pm 95$ \\
$\mathrm{OCS}$ & 78 & $2325 \pm 95$ \\
$\mathrm{NH}_{3}$ & 91 & $2715 \pm 105$ \\
$\mathrm{CS}_{2}$ & 95 & $2832 \pm 105$ \\
$\mathrm{SO}_{2}$ & 101 & $3010 \pm 110$ \\
$\mathrm{CH}_{3} \mathrm{CN}$ & 127 & $3790 \pm 130$ \\
$\mathrm{CH}_{3} \mathrm{OH}$ & 128 & $3820 \pm 135$ \\
$\mathrm{HCOOH}$ & 152 & $4532 \pm 150$ \\
\hline
\end{tabular}

Notes.

${ }^{\mathrm{a}}$ First desorption peak.

${ }^{\mathrm{b}}$ Second desorption peak.

category are $\mathrm{H}_{2} \mathrm{O}$-like, showing a desorption behavior similar to $\mathrm{H}_{2} \mathrm{O}$. Included in this category are $\mathrm{NH}_{3}, \mathrm{CH}_{3} \mathrm{OH}$, and $\mathrm{HCOOH}$. Different from the most volatile species, these $\mathrm{H}_{2} \mathrm{O}$-like species are unable to diffuse easily, and it is likely that these species bind strongly to the $\mathrm{H}_{2} \mathrm{O}$ molecules. Finally, we have the intermediate species category. In this category, we can find $\mathrm{H}_{2} \mathrm{~S}$, OCS, $\mathrm{CO}_{2}, \mathrm{C}_{2} \mathrm{H}_{2}, \mathrm{SO}_{2}, \mathrm{CS}_{2}$, and $\mathrm{CH}_{3} \mathrm{CN}$. When desorbing from water ice, these species present a volcano desorption and co-desorption peak, indicating that they are trapped in the water ice. Here, we have estimated the binding energies based on the results presented in Collings et al. (2004) for the deposition of each of these species on a $\mathrm{H}_{2} \mathrm{O}$ substrate. Our estimation is very simple and was done as follows:

$$
E_{\text {bind }, \mathrm{X}}=\frac{T_{\text {des, } \mathrm{X}}}{T_{\text {des, } \mathrm{H}_{2} \mathrm{O}}} \times E_{\text {bind, } \mathrm{H}_{2} \mathrm{O}},
$$

where $T_{\text {des, } \mathrm{X}}$ is the desorption temperature of species $\mathrm{X}$ deposited on a $\mathrm{H}_{2} \mathrm{O}$ film, $T_{\text {des, } \mathrm{H}_{2} \mathrm{O}}$ is the desorption temperature of $\mathrm{H}_{2} \mathrm{O}$, and $E_{\text {bind, } \mathrm{H}_{2} \mathrm{O}}$ is the binding energy of $\mathrm{H}_{2} \mathrm{O}$. The binding energy of water has been determined for a range of different substrates and preparation methods for the water ice. Here we chose $E_{\text {bind, } \mathrm{H}_{2} \mathrm{O}}=4800 \mathrm{~K}$, which is the result of a compilation of the amorphous water results (Sandford \& Allamandola 1988; Fraser et al. 2001; Brown \& Bolina 2007; Collings et al. 2015) taking into account the prefactor used in the gas-grain model. It agrees closely with the water binding energy to amorphous silicate (Dulieu et al. 2013). The values of $T_{\text {des, } \mathrm{H}_{2} \mathrm{O}}$ and $T_{\text {des, } \mathrm{X}}$ were obtained by visual inspection of Figure 2 of Collings et al. (2004), where $T_{\text {des, } \mathrm{H}_{2} \mathrm{O}}$ was estimated to be $161 \mathrm{~K}$ and $T_{\mathrm{des}, \mathrm{X}}$ varies according to the species. Since our approach results in an inherent error in the estimated binding energy, we applied an uncertainty of $3.5 \%$ to values in Table 2. As can be seen from Table 1, experimental binding energies for stable species typically have an error around $1 \%$.

The values we estimated for $E_{\mathrm{bind}, \mathrm{CO}}, E_{\mathrm{bind}, \mathrm{CO}_{2}}$, and $E_{\mathrm{bind}, \mathrm{O}_{2}}$ are in close agreement with those found by Noble et al. (2012a) for different substrates. Our estimations for $E_{\text {bind, } \mathrm{N}_{2}}$, based on Collings et al. (2004) using the first peak, are similar to those found in other studies (Öberg et al. 2005; Fuchs et al. 2006;
Collings et al. 2015). The value found by Smith et al. (2016) is much larger, but this can be explained by the much larger preexponential factor that they obtained in their fit. If this is accounted for by assuming the same desorption rate at the peak temperature $(\approx 25 \mathrm{~K})$, the binding energy corresponds to $990 \mathrm{~K}$, which is in closer agreement with our estimation from Collings et al. (2004). The reported binding energies for carbon monoxide, on the other hand, show a large deviation. Öberg et al. (2005), for instance, found $E_{\text {bind,CO }}=855 \mathrm{~K}$ based on experiments on multilayers of pure $\mathrm{CO}$ ice, while the computational binding energies of $\mathrm{CO}$ on various water substrates ranged between $1555 \mathrm{~K}$ and $1700 \mathrm{~K}$ in Karssemeijer \& Cuppen (2014). Other experimental studies (Sandford et al. 1988; Collings et al. 2003, 2015; Fuchs et al. 2006; Acharyya et al. 2007; Noble et al. 2012a; Smith et al. 2016) obtained values within these extremes. Our recommended value is based on Noble et al. (2012a), which is representative of CO on a water ice surface in the submonolayer regime. Additionally, we use a relatively high value of $250 \mathrm{~K}$ for the uncertainty to account for the large distribution of binding sites found in this study.

The binding energies and the corresponding uncertainties used here are summarized in Table 3. Experimental binding energy values are used for the following species: $\mathrm{C}_{2} \mathrm{H}_{2}$, $\mathrm{CH}_{3} \mathrm{CN}, \mathrm{CH}_{3} \mathrm{OH}, \mathrm{CH}_{4}, \mathrm{CO}, \mathrm{CO}_{2}, \mathrm{H}_{2}, \mathrm{H}_{2} \mathrm{CO}, \mathrm{H}_{2} \mathrm{O}, \mathrm{H}_{2} \mathrm{~S}$, $\mathrm{HCOOH}, \mathrm{N}_{2}, \mathrm{NH}_{3}, \mathrm{O}, \mathrm{O}_{2}, \mathrm{OCS}, \mathrm{OH}$, and $\mathrm{SO}_{2}$. He \& Vidali (2014) could only constrain the binding energy of $\mathrm{OH}$ to fall between $1656 \mathrm{~K}$ and $4760 \mathrm{~K}$. We used here the average with an uncertainty to cover this range. For $\mathrm{H}_{2}$, experiments deliver a large spread in the results. We chose to use here the intermediate value $E_{\text {bind, } \mathrm{H}_{2}}=500 \mathrm{~K}$, which is very similar to the value found by Acharyya (2014) using TPD experiments. The value for $\mathrm{CH}_{4}$ is based on Smith et al. (2016), who found a value of $1370 \mathrm{~K}$ with a high prefactor. Applying a prefactor of $10^{12} \mathrm{~s}^{-1}$, which is more representative of the prefactor typically used in gas-grain codes, and assuming the same desorption rate at the peak intensity ( $35 \mathrm{~K}$ ), a binding energy of $1130 \mathrm{~K}$ is obtained. We use an intermediate value of $1250 \mathrm{~K}$ with an uncertainty of $120 \mathrm{~K}$. The same approach is used for $\mathrm{C}_{2} \mathrm{H}_{4}$. Table 3 also shows the binding energies recommended by the UMIST ${ }^{4}$ Database for Astrochemistry UDfA (McElroy et al. 2013), which is commonly used. It is clear that for most of the species, the values of binding energies recommended by the present work and by the UDfA present large differences. These differences are better visualized in Figure 1, where the values of binding energies recommended by this work and by the UDfA database are plotted in a common diagram for a selection of species. For some species, these differences reflect a different choice in substrate. For other cases, the UDfA list simply predates the experimental work on which our value is based.

For all other species, we adopt the initial list from Hasegawa \& Herbst (1993a), which in turn is based on previous works (Allen \& Robinson 1977; Tielens \& Allamandola 1987), Aikawa et al. (1996), and Garrod \& Herbst (2006). For these cases, an uncertainty of $500 \mathrm{~K}$ has been used when the value is higher than $1000 \mathrm{~K}$; otherwise, the uncertainty is set to half of the binding energy.

\footnotetext{
http://www.udfa.net
} 
Table 3

Molecular Binding Energies

\begin{tabular}{|c|c|c|c|c|c|c|c|c|}
\hline \multicolumn{3}{|c|}{$\overline{E_{\text {bind }}(\mathrm{K})}$} & \multicolumn{3}{|c|}{$\overline{E_{\text {bind }}(\mathrm{K})}$} & \multicolumn{3}{|c|}{$E_{\text {bind }}(\mathrm{K})$} \\
\hline Species & This work & $\overline{\text { UMIST }}$ & Species & This work & $\overline{\text { UMIST }}$ & Species & This work & UMIST \\
\hline $\mathrm{C}$ & $715 \pm 360^{\mathrm{a}}$ & 800 & $\mathrm{CH}_{2} \mathrm{OHCHO}$ & $6680 \pm 500$ & & $\mathrm{HNO}$ & $1510 \pm 500^{\mathrm{b}}$ & 2050 \\
\hline $\mathrm{C}_{2}$ & $1085 \pm 500^{\mathrm{a}}$ & 1600 & $\mathrm{CH}_{2} \mathrm{OHCO}$ & $6230 \pm 500$ & & $\mathrm{HNOH}$ & $5230 \pm 500$ & \\
\hline $\mathrm{C}_{2} \mathrm{H}$ & $1330 \pm 500^{\mathrm{a}}$ & 2137 & $\mathrm{CH}_{2} \mathrm{PH}$ & $1200 \pm 500$ & & HNSi & $1100 \pm 500$ & 1100 \\
\hline $\mathrm{C}_{2} \mathrm{H}_{2}$ & $2090 \pm 85^{\mathrm{c}}$ & 2587 & $\mathrm{CH}_{3}$ & $1040 \pm 500^{\mathrm{a}}$ & 1175 & HOCN & $2850 \pm 500$ & \\
\hline $\mathrm{C}_{2} \mathrm{H}_{3}$ & $1760 \pm 500^{\mathrm{b}}$ & 3037 & $\mathrm{CH}_{3} \mathrm{C}_{3} \mathrm{~N}$ & $3880 \pm 500^{\mathrm{b}}$ & 6480 & HONC & $2850 \pm 500$ & \\
\hline $\mathrm{C}_{2} \mathrm{H}_{4}$ & $2010 \pm 500^{\mathrm{b}}$ & 3487 & $\mathrm{CH}_{3} \mathrm{C}_{4} \mathrm{H}$ & $3830 \pm 500^{\mathrm{b}}$ & 5887 & HPO & $1200 \pm 500$ & \\
\hline $\mathrm{C}_{2} \mathrm{H}_{4} \mathrm{CN}$ & $5930 \pm 500$ & & $\mathrm{CH}_{3} \mathrm{C}_{5} \mathrm{~N}$ & $5080 \pm 500^{\mathrm{b}}$ & 7880 & HS & $1350 \pm 500^{\mathrm{a}}$ & 1500 \\
\hline $\mathrm{C}_{2} \mathrm{H}_{5}$ & $2110 \pm 500^{\mathrm{b}}$ & 3937 & $\mathrm{CH}_{3} \mathrm{C}_{6} \mathrm{H}$ & $5030 \pm 500^{\mathrm{b}}$ & 7487 & $\mathrm{HS}_{2}$ & $2300 \pm 500^{\mathrm{b}}$ & 2650 \\
\hline $\mathrm{C}_{2} \mathrm{H}_{5} \mathrm{CN}$ & $6380 \pm 500$ & & $\mathrm{CH}_{3} \mathrm{C}_{7} \mathrm{~N}$ & $6290 \pm 500^{\mathrm{b}}$ & 9480 & $\mathrm{HCl}$ & $900 \pm 450$ & 900 \\
\hline $\mathrm{C}_{2} \mathrm{H}_{5} \mathrm{OH}$ & $3470 \pm 500^{\mathrm{b}}$ & 5200 & $\mathrm{CH}_{3} \mathrm{CCH}$ & $4290 \pm 500$ & 2470 & $\mathrm{~F}$ & $450 \pm 225$ & \\
\hline $\mathrm{C}_{2} \mathrm{H}_{6}$ & $2183 \pm 310^{\mathrm{d}}$ & 2300 & $\mathrm{CH}_{3} \mathrm{CHCH}_{2}$ & $5190 \pm 500$ & & $\mathrm{Fe}$ & $3750 \pm 500^{\mathrm{a}}$ & 4200 \\
\hline $\mathrm{C}_{2} \mathrm{~N}$ & $2010 \pm 500^{b}$ & 2400 & $\mathrm{CH}_{3} \mathrm{CHO}$ & $2870 \pm 500^{\mathrm{b}}$ & 3800 & $\mathrm{Mg}$ & $4750 \pm 500^{\mathrm{a}}$ & 5300 \\
\hline $\mathrm{C}_{2} \mathrm{O}$ & $2010 \pm 500^{\mathrm{b}}$ & 1950 & $\mathrm{CH}_{3} \mathrm{CN}$ & $3790 \pm 130^{c}$ & 4680 & $\mathrm{~N}$ & $715 \pm 358^{a}$ & 800 \\
\hline $\mathrm{C}_{2} \mathrm{~S}$ & $2500 \pm 500^{\mathrm{b}}$ & & $\mathrm{CH}_{3} \mathrm{CO}$ & $2320 \pm 500$ & & $\mathrm{~N}_{2}$ & $990 \pm 100^{\mathrm{d}}$ & 790 \\
\hline $\mathrm{C}_{3}$ & $2010 \pm 500^{b}$ & 2400 & $\mathrm{CH}_{3} \mathrm{COCH}_{3}$ & $3300 \pm 500$ & & $\mathrm{~N}_{2} \mathrm{O}$ & $2400 \pm 500$ & 2400 \\
\hline $\mathrm{C}_{3} \mathrm{H}$ & $2270 \pm 500^{\mathrm{b}}$ & 2937 & $\mathrm{CH}_{3} \mathrm{COOH}$ & $6300 \pm 500$ & & $\mathrm{NCCN}$ & $1300 \pm 500$ & 1300 \\
\hline $\mathrm{C}_{3} \mathrm{H}_{2}$ & $2110 \pm 500^{\mathrm{b}}$ & 3387 & $\mathrm{CH}_{3} \mathrm{NH}$ & $1760 \pm 500^{\mathrm{b}}$ & 3553 & $\mathrm{NH}$ & $542 \pm 270^{\mathrm{a}}$ & 2378 \\
\hline $\mathrm{C}_{3} \mathrm{~N}$ & $2720 \pm 500^{b}$ & 3200 & $\mathrm{CH}_{3} \mathrm{NH}_{2}$ & $5130 \pm 500$ & & $\mathrm{NH}_{2}$ & $770 \pm 385^{\mathrm{a}}$ & 3956 \\
\hline $\mathrm{C}_{3} \mathrm{O}$ & $2520 \pm 500^{b}$ & 2750 & $\mathrm{CH}_{3} \mathrm{O}$ & $2655 \pm 500$ & 5080 & $\mathrm{NH}_{2} \mathrm{CHO}$ & $5560 \pm 500$ & 5556 \\
\hline $\mathrm{C}_{3} \mathrm{P}$ & $1650 \pm 500$ & & $\mathrm{CH}_{3} \mathrm{OCH}_{3}$ & $2820 \pm 500^{\mathrm{b}}$ & 3300 & $\mathrm{NH}_{2} \mathrm{CN}$ & $1200 \pm 500$ & 1200 \\
\hline $\mathrm{C}_{3} \mathrm{~S}$ & $3000 \pm 500^{\mathrm{b}}$ & 3500 & $\mathrm{CH}_{3} \mathrm{OH}$ & $3820 \pm 135^{\mathrm{c}}$ & 4930 & $\mathrm{NH}_{2} \mathrm{CO}$ & $5110 \pm 500$ & \\
\hline $\mathrm{C}_{4}$ & $2420 \pm 500^{\mathrm{b}}$ & 3200 & $\mathrm{CH}_{4}$ & $1250 \pm 120^{\mathrm{d}}$ & 1090 & $\mathrm{NH}_{2} \mathrm{OH}$ & $2770 \pm 500^{\mathrm{b}}$ & 6806 \\
\hline $\mathrm{C}_{4} \mathrm{H}$ & $2670 \pm 500^{\mathrm{b}}$ & 3737 & $\mathrm{CN}$ & $1355 \pm 500^{\mathrm{a}}$ & 1600 & $\mathrm{NH}_{3}$ & $2715 \pm 105^{c}$ & 5534 \\
\hline $\mathrm{C}_{4} \mathrm{H}_{2}$ & $2920 \pm 500^{b}$ & 4187 & $\mathrm{CNO}$ & $2400 \pm 500$ & & NO & $1085 \pm 500^{\mathrm{a}}$ & 1600 \\
\hline $\mathrm{C}_{4} \mathrm{H}_{3}$ & $2970 \pm 500^{\mathrm{b}}$ & 4637 & $\mathrm{CO}$ & $1100 \pm 250^{\mathrm{e}}$ & 1150 & $\mathrm{NO}_{2}$ & $2400 \pm 500$ & 2400 \\
\hline $\mathrm{C}_{4} \mathrm{H}_{6}$ & $5990 \pm 500$ & 5987 & $\mathrm{CO}_{2}$ & $2267 \pm 70^{\mathrm{e}}$ & 2990 & NS & $1800 \pm 500^{\mathrm{a}}$ & 1900 \\
\hline $\mathrm{C}_{4} \mathrm{~N}$ & $3220 \pm 500^{\mathrm{b}}$ & 4000 & $\mathrm{COOCH}_{3}$ & $3650 \pm 500$ & & $\mathrm{Na}$ & $10600 \pm 500^{\mathrm{a}}$ & 11800 \\
\hline $\mathrm{C}_{4} \mathrm{P}$ & $1950 \pm 500$ & & $\mathrm{COOH}$ & $5120 \pm 500$ & & $\mathrm{O}$ & $1660 \pm 60^{\mathrm{f}}$ & 800 \\
\hline $\mathrm{C}_{4} \mathrm{~S}$ & $3500 \pm 500^{\mathrm{b}}$ & 4300 & $\mathrm{CP}$ & $1050 \pm 500$ & & $\mathrm{O}_{2}$ & $898 \pm 30^{\mathrm{e}}$ & 1000 \\
\hline $\mathrm{C}_{5}$ & $3220 \pm 500^{b}$ & 4000 & $\mathrm{CS}$ & $1800 \pm 500^{\mathrm{a}}$ & 1900 & $\mathrm{O}_{2} \mathrm{H}$ & $1510 \pm 500^{\mathrm{b}}$ & 3650 \\
\hline $\mathrm{C}_{5} \mathrm{H}$ & $3470 \pm 500^{\mathrm{b}}$ & 4537 & $\mathrm{Cl}$ & $850 \pm 425$ & 850 & $\mathrm{O}_{3}$ & $2100 \pm 100^{\mathrm{g}}$ & 1800 \\
\hline $\mathrm{C}_{5} \mathrm{H}_{2}$ & $3730 \pm 500^{\mathrm{b}}$ & 4987 & $\mathrm{ClO}$ & $1250 \pm 500$ & 1250 & $\mathrm{OCN}$ & $1805 \pm 500^{\mathrm{a}}$ & 2400 \\
\hline $\mathrm{C}_{5} \mathrm{~N}$ & $3930 \pm 500^{\mathrm{b}}$ & 4800 & $\mathrm{H}$ & $650 \pm 100^{\mathrm{h}}$ & 600 & OCS & $2325 \pm 95^{\mathrm{c}}$ & 2888 \\
\hline $\mathrm{C}_{6}$ & $3620 \pm 500^{\mathrm{b}}$ & 4800 & $\mathrm{H}_{2}$ & $500 \pm 100^{\mathrm{i}}$ & 430 & $\mathrm{OH}$ & $3210 \pm 1550^{\mathrm{f}}$ & 2850 \\
\hline $\mathrm{C}_{6} \mathrm{H}$ & $3880 \pm 500^{\mathrm{b}}$ & 5337 & $\mathrm{H}_{2} \mathrm{CCC}$ & $2110 \pm 500$ & 2110 & $\mathrm{P}$ & $750 \pm 375$ & \\
\hline $\mathrm{C}_{6} \mathrm{H}_{2}$ & $4130 \pm 500^{\mathrm{b}}$ & 5787 & $\mathrm{H}_{2} \mathrm{CN}$ & $2400 \pm 500$ & 2400 & $\mathrm{PH}$ & $800 \pm 400$ & \\
\hline $\mathrm{C}_{6} \mathrm{H}_{6}$ & $7590 \pm 500$ & 7587 & $\mathrm{H}_{2} \mathrm{CO}$ & $3260 \pm 60^{\mathrm{e}}$ & 2050 & $\mathrm{PH}_{2}$ & $850 \pm 425$ & \\
\hline $\mathrm{C}_{7}$ & $4430 \pm 500^{\mathrm{b}}$ & 5600 & $\mathrm{H}_{2} \mathrm{CS}$ & $2025 \pm 500^{\mathrm{a}}$ & 2700 & $\mathrm{PN}$ & $1100 \pm 500$ & \\
\hline $\mathrm{C}_{7} \mathrm{H}$ & $4680 \pm 500^{\mathrm{b}}$ & 6137 & $\mathrm{H}_{2} \mathrm{O}$ & $4800 \pm 100$ & 4800 & PO & $1150 \pm 500$ & \\
\hline $\mathrm{C}_{7} \mathrm{H}_{2}$ & $4930 \pm 500^{b}$ & 6587 & $\mathrm{H}_{2} \mathrm{O}_{2}$ & $6000 \pm 100^{\mathrm{g}}$ & 5700 & S & $985 \pm 495^{\mathrm{a}}$ & 1100 \\
\hline $\mathrm{C}_{7} \mathrm{~N}$ & $5130 \pm 500^{\mathrm{b}}$ & 6400 & $\mathrm{H}_{2} \mathrm{~S}$ & $2290 \pm 90^{c}$ & 2743 & $\mathrm{~S}_{2}$ & $2000 \pm 500^{\mathrm{b}}$ & 2200 \\
\hline $\mathrm{C}_{8}$ & $4830 \pm 500^{b}$ & 6400 & $\mathrm{H}_{2} \mathrm{~S}_{2}$ & $2600 \pm 500^{b}$ & 3100 & $\mathrm{Si}$ & $2400 \pm 500^{\mathrm{a}}$ & 2700 \\
\hline $\mathrm{C}_{8} \mathrm{H}$ & $5080 \pm 500^{\mathrm{b}}$ & 6937 & $\mathrm{H}_{2} \mathrm{SiO}$ & $1200 \pm 500$ & 1200 & $\mathrm{SiC}$ & $3150 \pm 500^{\mathrm{a}}$ & 3500 \\
\hline $\mathrm{C}_{8} \mathrm{H}_{2}$ & $5340 \pm 500^{\mathrm{b}}$ & 7387 & $\mathrm{HC}_{2} \mathrm{O}$ & $2010 \pm 500^{\mathrm{b}}$ & 2400 & $\mathrm{SiC}_{2}$ & $1300 \pm 500$ & 1300 \\
\hline $\mathrm{C}_{9}$ & $5640 \pm 500^{\mathrm{b}}$ & 7200 & $\mathrm{HC}_{2} \mathrm{P}$ & $1400 \pm 500$ & & $\mathrm{SiC}_{2} \mathrm{H}$ & $1350 \pm 500$ & 1350 \\
\hline $\mathrm{C}_{9} \mathrm{H}$ & $5890 \pm 500^{\mathrm{b}}$ & 7737 & $\mathrm{HC}_{3} \mathrm{~N}$ & $2685 \pm 500^{\mathrm{a}}$ & 4580 & $\mathrm{SiC}_{2} \mathrm{H}_{2}$ & $1400 \pm 500$ & 1400 \\
\hline $\mathrm{C}_{9} \mathrm{H}_{2}$ & $6140 \pm 500^{\mathrm{b}}$ & 8187 & $\mathrm{HC}_{5} \mathrm{~N}$ & $4180 \pm 500^{\mathrm{b}}$ & 6180 & $\mathrm{SiC}_{3}$ & $1600 \pm 500$ & \\
\hline $\mathrm{C}_{9} \mathrm{~N}$ & $6340 \pm 500^{\mathrm{b}}$ & 8000 & $\mathrm{HC}_{7} \mathrm{~N}$ & $5390 \pm 500^{\mathrm{b}}$ & 7780 & $\mathrm{SiC}_{3} \mathrm{H}$ & $1650 \pm 500$ & 1650 \\
\hline $\mathrm{C}_{10}$ & $8000 \pm 500$ & 8000 & $\mathrm{HC}_{9} \mathrm{~N}$ & $6590 \pm 500^{\mathrm{b}}$ & 9380 & $\mathrm{SiC}_{4}$ & $1900 \pm 500$ & 1900 \\
\hline $\mathrm{C}_{10} \mathrm{H}$ & $8540 \pm 500$ & & $\mathrm{HCCN}$ & $2270 \pm 500^{\mathrm{b}}$ & 3780 & $\mathrm{SiCH}_{2}$ & $1100 \pm 500$ & 1100 \\
\hline $\mathrm{C}_{10} \mathrm{H}_{2}$ & $8990 \pm 500$ & & $\mathrm{HCN}$ & $1580 \pm 500^{\mathrm{a}}$ & 2050 & $\mathrm{SiCH}_{3}$ & $1150 \pm 500$ & 1150 \\
\hline $\mathrm{C}_{11}$ & $8800 \pm 500$ & & HCNO & $2850 \pm 500$ & & $\mathrm{SiH}$ & $2620 \pm 500^{\mathrm{a}}$ & 3150 \\
\hline $\mathrm{CCP}$ & $1350 \pm 500$ & & $\mathrm{HCO}$ & $1355 \pm 500^{\mathrm{a}}$ & 1600 & $\mathrm{SiH}_{2}$ & $3190 \pm 500^{\mathrm{b}}$ & 3600 \\
\hline $\mathrm{CCl}$ & $1150 \pm 500$ & 1150 & $\mathrm{HCOOCH}_{3}$ & $4000 \pm 500$ & 4000 & $\mathrm{SiH}_{3}$ & $3440 \pm 500^{\mathrm{b}}$ & 4050 \\
\hline $\mathrm{CH}$ & $590 \pm 295^{\mathrm{a}}$ & 925 & $\mathrm{HCOOH}$ & $4532 \pm 150^{\mathrm{c}}$ & 5000 & $\mathrm{SiH}_{4}$ & $3690 \pm 500^{\mathrm{b}}$ & 4500 \\
\hline $\mathrm{CH}_{2}$ & $860 \pm 430^{\mathrm{a}}$ & 1050 & $\mathrm{HCP}$ & $1100 \pm 500$ & & $\mathrm{SiN}$ & $3500 \pm 500$ & 3500 \\
\hline $\mathrm{CH}_{2} \mathrm{CCH}$ & $3840 \pm 500$ & & HCS & $2000 \pm 500^{\mathrm{b}}$ & 2350 & SiNC & $1350 \pm 500$ & 1350 \\
\hline $\mathrm{CH}_{2} \mathrm{CCH}_{2}$ & $4290 \pm 500$ & & $\mathrm{HCSi}$ & $1050 \pm 500$ & 1050 & $\mathrm{SiO}$ & $3150 \pm 500^{\mathrm{a}}$ & 3500 \\
\hline $\mathrm{CH}_{2} \mathrm{CHCCH}$ & $5090 \pm 500$ & & $\mathrm{He}$ & $100 \pm 50^{\mathrm{b}}$ & 100 & $\mathrm{SiO}_{2}$ & $4300 \pm 500$ & 4300 \\
\hline $\mathrm{CH}_{2} \mathrm{CHCN}$ & $5480 \pm 500$ & 5480 & $\mathrm{HF}$ & $500 \pm 250$ & & $\mathrm{SiS}$ & $3400 \pm 500^{\mathrm{a}}$ & 3800 \\
\hline $\mathrm{CH}_{2} \mathrm{CN}$ & $2470 \pm 500^{\mathrm{b}}$ & 4230 & $\mathrm{HNC}$ & $1510 \pm 500^{\mathrm{b}}$ & 2050 & SO & $1800 \pm 500^{\mathrm{a}}$ & 2600 \\
\hline $\mathrm{CH}_{2} \mathrm{CO}$ & $2520 \pm 500^{\mathrm{b}}$ & 2200 & $\mathrm{HNC}_{3}$ & $4580 \pm 500$ & 4580 & $\mathrm{SO}_{2}$ & $3010 \pm 110^{c}$ & 5330 \\
\hline $\mathrm{CH}_{2} \mathrm{NH}$ & $1560 \pm 500$ & 3428 & HNCHO & $3980 \pm 500$ & & & & \\
\hline
\end{tabular}


Table 3

(Continued)

\begin{tabular}{|c|c|c|c|c|c|c|c|c|}
\hline \multicolumn{3}{|c|}{$E_{\text {bind }}(\mathrm{K})$} & \multicolumn{3}{|c|}{$E_{\text {bind }}(\mathrm{K})$} & \multicolumn{3}{|c|}{$E_{\text {bind }}(\mathrm{K})$} \\
\hline Species & This work & UMIST & Species & This work & $\overline{\text { UMIST }}$ & Species & This work & UMIST \\
\hline$\overline{\mathrm{CH}_{2} \mathrm{OH}}$ & $2170 \pm 500^{\mathrm{b}}$ & 5084 & HNCO & $2270 \pm 500^{\mathrm{b}}$ & 2850 & & & \\
\hline
\end{tabular}

Notes. Species for which experimental values have been derived are highlighted.

${ }^{a}$ Average between Hasegawa \& Herbst (1993a) and Aikawa et al. (1996) values.

${ }^{\mathrm{b}}$ Hasegawa \& Herbst (1993a).

${ }^{\mathrm{c}}$ Estimated from Collings et al. (2004).

${ }^{\mathrm{d}}$ Smith et al. (2016), with a change in the prefactor.

e Noble et al. (2012a).

${ }^{\mathrm{f}} \mathrm{He}$ et al. (2014), He \& Vidali (2014).

${ }^{\mathrm{g}}$ Dulieu et al. (2013).

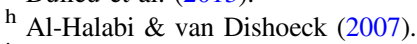

${ }^{\mathrm{i}}$ Similar to Acharyya (2014).

${ }^{j}$ Average between Tielens \& Allamandola (1987) and Aikawa et al. (1996) values.

(This table is available in machine-readable form.)

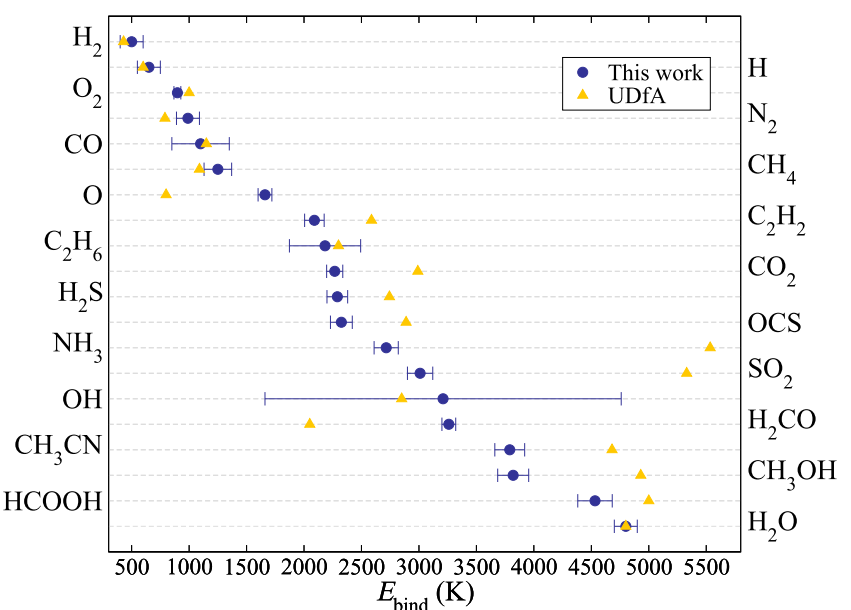

Figure 1. Comparison of binding energies recommended by the present work (blue circles) based on an extensive literature review and those binding energies currently recommended by UDfA (yellow triangles). Most of species shown here are those for which experimental values have been derived. The dashed gray line is a guide to the eye to identify the correspondent species.

\section{Chemical Model}

We have simulated the time-dependent gas-grain chemistry of a homogeneous dark cloud with constant physical conditions. This is a two-phase chemistry model, since it treats the gas and solid phases, although without using the location information of ice species within the ice mantle. The model, therefore, does not differentiate between reactions between species located on the ice surface and in the bulk mantle; however, grain-surface reactions are limited to occur within two monolayers worth of material in the ice mantle only. Here, we give a brief explanation of the model. For more details, we refer to Walsh et al. (2015) and references therein.

\subsection{Gas-Grain Network and Physical Conditions}

The gas-phase network is the UDfA Database for Astrochemistry (McElroy et al. 2013), known as RATE12. This network does not account for three-body reactions, since these are not important at the density used here. Photoreactions and direct cosmic-ray ionization are included. The cosmic-ray ionization rate $(\zeta)$ used is $1.3 \times 10^{-17} \mathrm{~s}^{-1}$ (Indriolo et al. 2015).

The solid-phase chemistry is based on the Ohio State University (OSU) network ${ }^{5}$ (Garrod et al. 2008), which includes gas-grain interactions such as desorption and adsorption processes, and grain-surface chemistry. We neglect cosmic-ray-induced thermal desorption and reactive desorption because the rates for these processes remain very uncertain compared with thermal desorption and photodesorption. The model also includes grain-cation recombination. We assume a spherical and compact grain with a radius of $0.1 \mu \mathrm{m}$ for simplicity. The grain abundance is fixed to $1.3 \times 10^{-12}$ with respect to $\mathrm{H}$ nuclei, and the density of grain-surface sites is $1.5 \times 10^{15} \mathrm{~cm}^{-2}$. The ratio between the diffusion barrier $\left(E_{\text {diff }}\right)$ and the molecular binding energy is assumed to be $E_{\text {diff }}=0.3 \times E_{\text {bind }}$. The value of $E_{\text {diff }} / E_{\text {bind }}$ is still under debate, and most modelers (Hasegawa et al. 1992; Ruffle \& Herbst 2000; Garrod \& Herbst 2006; Cuppen et al. 2009) have used values ranging from 0.3 to 0.8 , although the consensus is that for stable species, this ratio should be between 0.3 and 0.4 (Karssemeijer \& Cuppen 2014). Here, we chose 0.3, which is an optimistic value that allows the radicals in the grain mantle to diffuse with some efficiency at low temperatures.

In the subsequent sections, we present results for many thousands of simulations for a fixed set of physical conditions and input parameters, except for the set of molecular binding energies. The physical conditions used here are those typical for a dark cloud. The initial abundances are taken from McElroy et al. (2013), which are identical to those used in Garrod et al. (2009). These initial abundances follow the lower metallicity set from Graedel et al. (1982). The total $\mathrm{H}$ nuclei density is $2 \times 10^{4} \mathrm{~cm}^{-3}$, and the visual extinction $\left(A_{\mathrm{V}}\right)$ is $10 \mathrm{mag}$. Both gas and dust temperatures are $10 \mathrm{~K}$. All simulations were performed over a timescale of $10^{8}$ years, at which time steady state is expected to be reached. Table 4 summarizes the initial elemental abundances, while Table 5 summarizes the assumed physical parameters. This is a $0 \mathrm{D}$ model, which means that all physical parameters are kept constant during the simulations.

In each simulation, a value of the binding energy for each grain-surface species is chosen at random from a normal

\footnotetext{
5 http://faculty.virginia.edu/ericherb/
} 
Table 4

Initial Abundances with Respect to H Nuclei

\begin{tabular}{lc}
\hline \hline Species & Abundances \\
\hline $\mathrm{H}$ & $5.00(-05)$ \\
$\mathrm{H}_{2}$ & $5.00(-01)$ \\
$\mathrm{He}$ & $9.75(-02)$ \\
$\mathrm{C}$ & $1.40(-04)$ \\
$\mathrm{N}$ & $7.50(-05)$ \\
$\mathrm{O}$ & $3.20(-04)$ \\
$\mathrm{F}$ & $2.00(-08)$ \\
$\mathrm{Na}$ & $2.00(-09)$ \\
$\mathrm{Mg}$ & $7.00(-09)$ \\
$\mathrm{Si}$ & $8.00(-09)$ \\
$\mathrm{P}$ & $3.00(-09)$ \\
$\mathrm{S}$ & $8.00(-08)$ \\
$\mathrm{Cl}$ & $4.00(-09)$ \\
$\mathrm{Fe}$ & $3.00(-09)$ \\
$\mathrm{Grain}$ density & $1.30(-12)$ \\
\hline
\end{tabular}

Note. The notation $\alpha(\beta)$ stands for $\alpha \times 10^{\beta}$.

Table 5

Dark Cloud Physical Parameters

\begin{tabular}{lc}
\hline \hline$n_{\mathrm{H}}$ & $2 \times 10^{4} \mathrm{~cm}^{-3}$ \\
$T_{\text {dust }}$ & $10 \mathrm{~K}$ \\
$T_{\text {gas }}$ & $10 \mathrm{~K}$ \\
$A_{\mathrm{V}}$ & $10 \mathrm{mag}$ \\
$\zeta$ & $1.3 \times 10^{-17} \mathrm{~s}^{-1}$ \\
\hline
\end{tabular}

probability distribution. The mean value of the normal distribution is the binding energy and the uncertainties correspond to a $3 \sigma$ error. The diffusion rates for each species $i$ are then calculated using

$$
k_{\text {thermal }, i}=\nu \exp \left(-0.3 \frac{E_{\text {bind }, i}}{k_{\mathrm{B}} T}\right)
$$

and

$$
k_{\text {quantum }, i}=\nu \exp \left(-\frac{2 a}{\hbar} \sqrt{0.6 \mu E_{\mathrm{bind}, i}}\right)
$$

by assuming thermal or quantum processes. Quantum diffusion is only allowed for light species such as $\mathrm{H}$ and $\mathrm{H}_{2}$. In the equations above, $\nu, a$, and $\mu$ are the frequency, the barrier width, and the reduced mass, respectively. These equations show that the binding energies have strong influence on the diffusion rates. Typical values for $\nu$ are $10^{12} \mathrm{~s}^{-1}$, and we adopt a barrier width between surface sites of $1.5 \AA$.

\section{Results}

\subsection{Updating the Network}

The first set of models used the standard network from Drozdovskaya et al. (2014, 2015) and Walsh et al. (2015), who adopt the surface network from Garrod et al. (2008) and mainly focused on O-bearing complex organic molecules. We ran a thousand simulations using the standard network. For the key ice species, $\mathrm{CO}, \mathrm{HCO}, \mathrm{H}_{2} \mathrm{CO}, \mathrm{CH}_{3} \mathrm{OH}, \mathrm{HCOOH}, \mathrm{H}_{2} \mathrm{O}, \mathrm{CO}_{2}$, $\mathrm{CH}_{4}$, and $\mathrm{NH}_{3}$, we then calculated the Pearson correlation coefficient between the logarithm of the abundance at different times and the binding energy of each of the species listed in Table 3

$$
P(A, B)=\frac{\operatorname{cov}\left(\log (n(A)), E_{\mathrm{bind}}(B)\right)}{\sigma(\log (n(A))) \sigma\left(E_{\mathrm{bind}}(B)\right)},
$$

where cov stands for the covariance and $\sigma$ is the standard deviation. This coefficient gives a measure of linear correlation or anti-correlation between the logarithm of the ice abundance and the binding energy, and lies between -1 and 1 . We selected those species that have an absolute correlation coefficient equal to or larger than 0.3 , i.e., $|P| \geqslant 0.3$. The binding energy of HNO was found to correlate with the log of the abundance of several species, which is rather unexpected since HNO is not a direct precursor to any of these species. $\mathrm{HNO}$ acts, however, as an efficient producer of $\mathrm{OH}$ radicals via the reaction

$$
\mathrm{HNO}+\mathrm{O} \rightarrow \mathrm{NO}+\mathrm{OH} .
$$

HNO is formed through the hydrogenation of NO. A dominant destruction reaction for $\mathrm{HNO}$ in our standard network is the reaction with $\mathrm{H}$ leading to $\mathrm{NO}$ and $\mathrm{H}_{2}$. $\mathrm{NO}$ catalyzes in this way the production of $\mathrm{OH}$ from $\mathrm{O}$ and $\mathrm{H}$ by making $\mathrm{H}$ temporarily unavailable for other reactions. However, laboratory experiments (Congiu et al. 2012; Fedoseev et al. 2012) show that hydrogenation of HNO leads predominantly to the formation of $\mathrm{NH}_{2} \mathrm{OH}$ through

$$
\mathrm{HNO}+\mathrm{H} \rightarrow \mathrm{H}_{2} \mathrm{NO}
$$

followed by

$$
\mathrm{H}_{2} \mathrm{NO}+\mathrm{H} \rightarrow \mathrm{NH}_{2} \mathrm{OH} .
$$

These reactions will take HNO out of the loop and block the formation of new $\mathrm{OH}$ radicals that can lead to the formation of $\mathrm{CH}_{3} \mathrm{OH}, \mathrm{HCOOH}, \mathrm{CO}_{2}$, and $\mathrm{H}_{2} \mathrm{O}$. We have therefore updated the network by adding the chemistry for $\mathrm{NH}_{2} \mathrm{OH}$, which includes the gas-phase chemistry of the neutrals and all intermediate ions. We have also included the chemistry for $\mathrm{NH}_{2} \mathrm{CHO}$ (formamide) and $\mathrm{CH}_{3} \mathrm{NH}_{2}$ (methylamine), both gas and surface reactions and also extracted from the OSU network (Garrod et al. 2008). This first set of simulations shows clearly the importance of new laboratory experiments to investigate chemical pathways that are not yet considered in most of astrochemical models but are fundamental to obtain more reliable results. The final network contains 8971 reactions connected by 689 species.

\subsection{Varying Binding Energies}

Using this new network, we performed 10,000 simulations selecting a set of binding energies at random from their normal distributions. Figure 2 shows the evolution of the ice abundance of some key ice species- $\mathrm{CO}, \mathrm{HCO}, \mathrm{H}_{2} \mathrm{CO}, \mathrm{CH}_{3} \mathrm{OH}, \mathrm{HCOOH}$, $\mathrm{H}_{2} \mathrm{O}, \mathrm{CO}_{2}, \mathrm{CH}_{4}$, and $\mathrm{NH}_{3}$. The abundance is taken with respect to $\mathrm{H}$ nuclei. Clearly, there is a large variation in steady-state abundance, especially for the species with $\mathrm{CO}$ as one of their precursors, but also in the time evolution of the abundances. To assess the origin of these differences, we again determined the Pearson correlation coefficient between the binding energies and the log of the abundance with respect to $\mathrm{H}$ nuclei. The binding energy of $\mathrm{H}_{2}$ was found to be the most strongly correlating parameter. This is evident in Figure 3, which shows the ice 


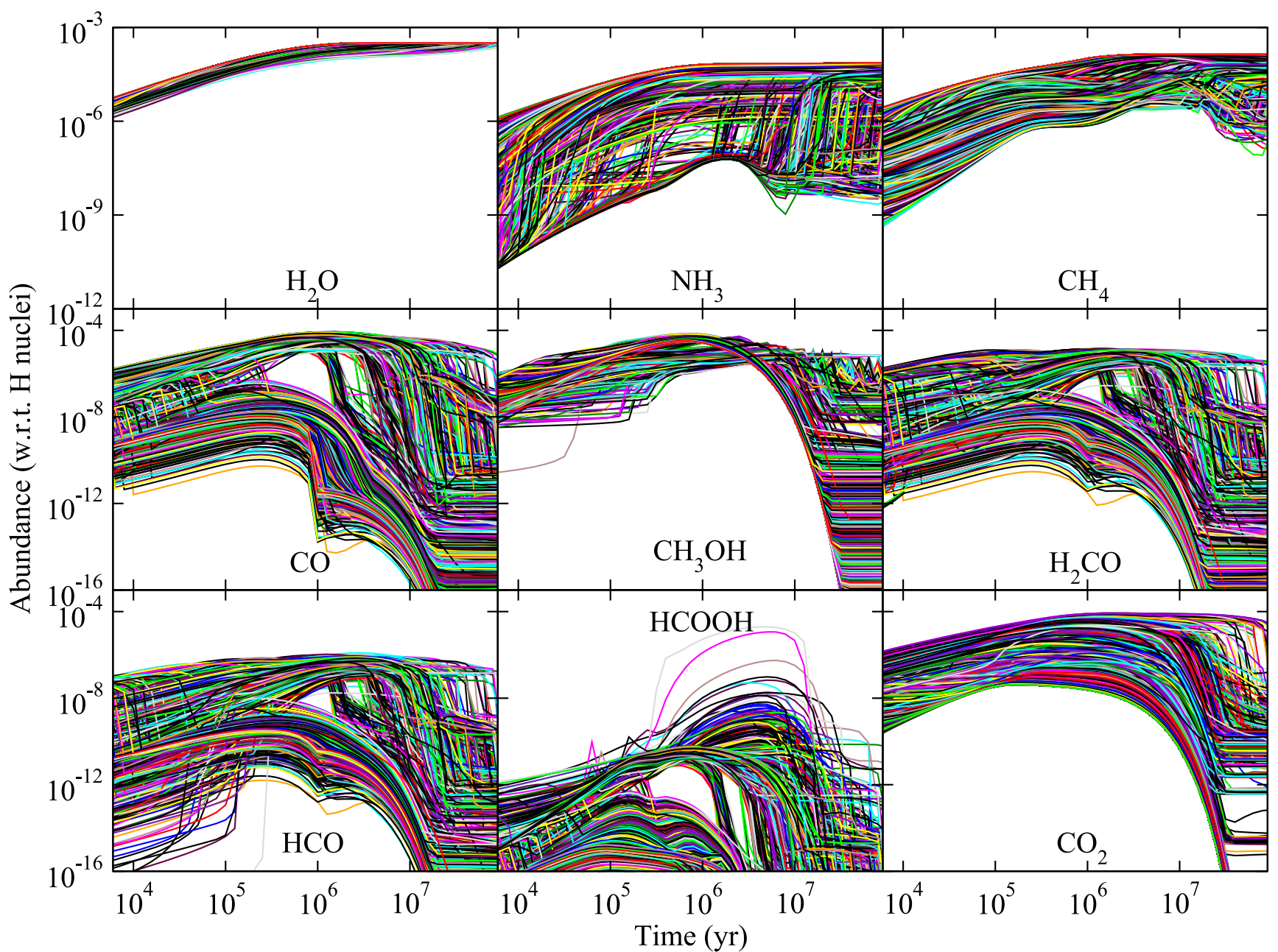

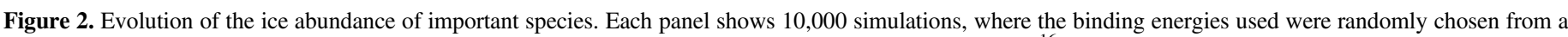
normal distribution. All abundances are shown with respect to $\mathrm{H}$ nuclei. Abundances much lower than $10^{-16}$ are negligible and are therefore not shown here.

abundance at three different times- $10^{5}$ (darker blue), $5 \times 10^{5}$ (darker yellow), and $10^{6}$ (darker green) years-as a function of $\mathrm{H}_{2}$ binding energy. There is a clear change in chemistry around $E_{\text {bind, } \mathrm{H}_{2}}=460 \mathrm{~K}$. For some species, this change occurs somewhat earlier than for others. Two groups can be identified according to similar general behavior: a group, including $\mathrm{CH}_{4}$ and $\mathrm{NH}_{3}$, which has a high abundance for high $E_{\text {bind, } \mathrm{H}_{2}}$, and a group with $\mathrm{CO}, \mathrm{HCO}, \mathrm{H}_{2} \mathrm{CO}, \mathrm{CO}_{2}$, and $\mathrm{HCOOH}$, which shows a sharp decrease with increasing $E_{\text {bind, } \mathrm{H}_{2}}$. The abundance of water and methanol is less sensitive to the value of $\mathrm{H}_{2}$ binding energy.

To determine the origin of this bifurcation, we have analyzed the two populations containing runs with $E_{\mathrm{bind}_{\mathrm{H}} \mathrm{H}_{2}}<460 \mathrm{~K}$ and $E_{\text {bind, } \mathrm{H}_{2}}>465 \mathrm{~K}$ separately. By identifying correlations between ice abundances and binding energies in both simulation populations separately, we hope to gain insight into the change in chemistry that is causing this effect. The discontinuity in temperature is intentional in order to isolate the two sets of results. The binding energy of $\mathrm{H}_{2}$ still correlates most frequently with the different ice abundances, mainly at later times. Other species whose binding energies appear to correlate strongly are $\mathrm{HCO}, \mathrm{H}, \mathrm{N}, \mathrm{CH}_{2}$, and $\mathrm{HNO}$.

Using these correlations together with information on the most important formation and destruction reactions for the ice species of interest, we extract the limited reaction network that explains the observed trends in the results from the full reaction network containing 2164 surface reactions. This network is depicted in Figure 4. The important precursors in this network are $\mathrm{NH}_{2}$ (formed from $\mathrm{N}$ ) and $\mathrm{C}$. With increasing $\mathrm{H}_{2}$ binding energies, the residence time of $\mathrm{H}_{2}$ on the surface increases, whereas the diffusion barrier for all cases is low enough for $\mathrm{H}_{2}$ to be mobile. For a sufficiently high $E_{\text {bind, } \mathrm{H}_{2}}, \mathrm{C}$ and $\mathrm{NH}_{2}$ react sequentially with $\mathrm{H}_{2}$ to form $\mathrm{CH}_{4}$ and $\mathrm{NH}_{3}$, respectively. These pathways are indicated in blue in the schematic. Water is predominantly made through

$$
\mathrm{OH}+\mathrm{H}_{2} \rightarrow \mathrm{H}_{2} \mathrm{O}+\mathrm{H},
$$

and its abundance therefore increases with $\mathrm{H}_{2}$ binding energy. The hydroxyl radical, $\mathrm{OH}$, can be formed in different ways. We found the dominant channel to be $\mathrm{H}+\mathrm{O}$. For low $\mathrm{H}_{2}$ binding energies,

$$
\mathrm{O}+\mathrm{HCO} \rightarrow \mathrm{CO}+\mathrm{OH}
$$

and

$$
\mathrm{H}+\mathrm{H}_{2} \mathrm{O}_{2} \rightarrow \mathrm{H}_{2} \mathrm{O}+\mathrm{OH}
$$

become important as well.

At some critical $\mathrm{H}_{2}$ binding energy, the $\mathrm{H}_{2}$ residence time becomes too short to be able to compete with oxidation reactions of both $\mathrm{C}$ and $\mathrm{NH}_{2}$, leading to the formation of $\mathrm{CO}$ and $\mathrm{HNO}$, 


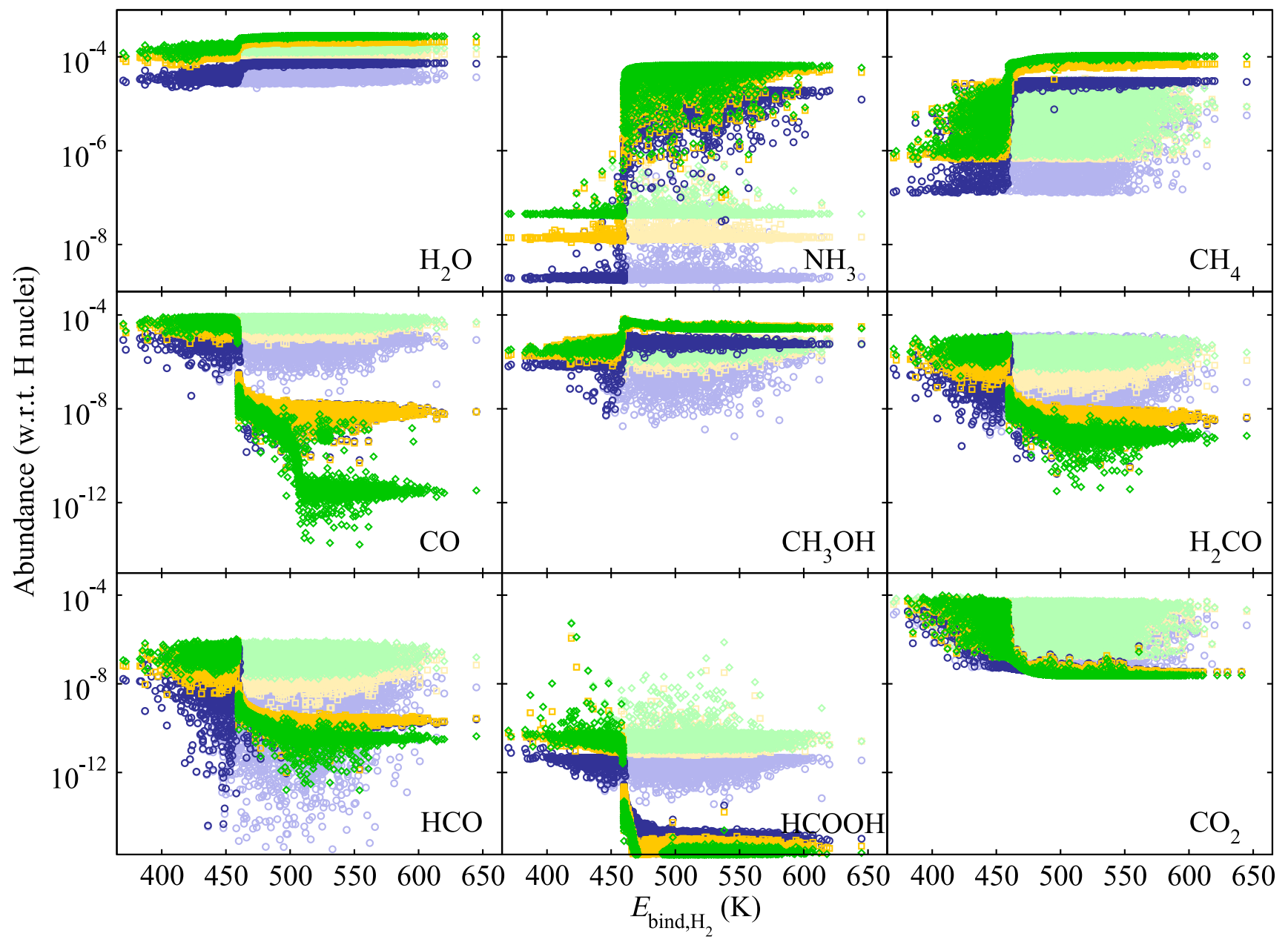

Figure 3. Abundance of ice species as a function of $\mathrm{H}_{2}$ binding energy. Each panel shows abundances from 10,000 simulations, each one derived from a different set of binding energies. Results for $10^{5}$ years are shown in blue, while results for $5 \times 10^{5}$ years are shown in yellow, and those for $10^{6}$ years are shown in green. All abundances are calculated with respect to $\mathrm{H}$ nuclei. Abundances much lower than $10^{-16}$ are negligible and are therefore not shown here. The darker colors represent the original model, whereas the lighter colors represent results using a model that reduces $\mathrm{H}_{2}$ freeze out.

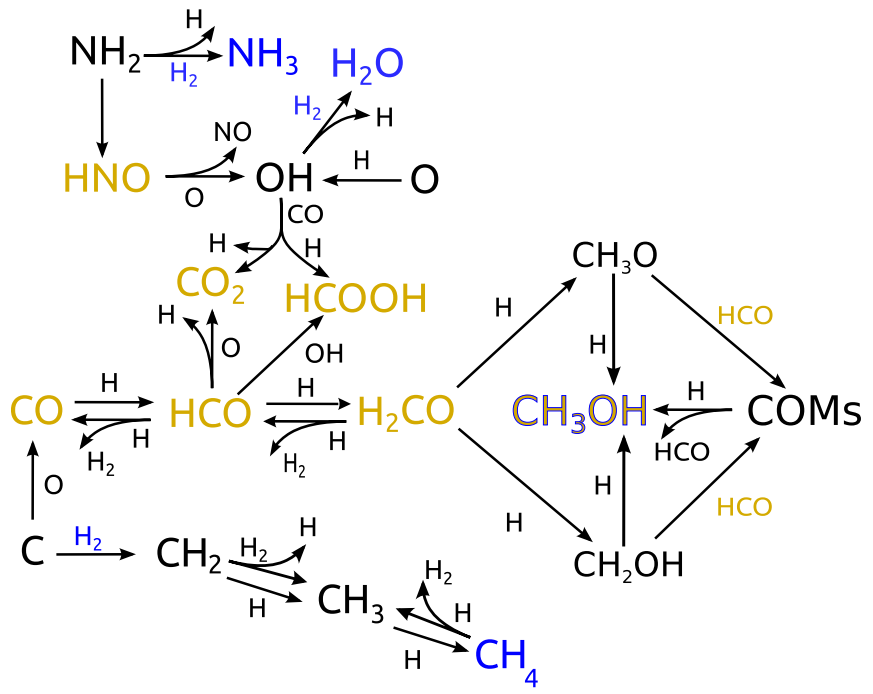

Figure 4. A limited surface network explaining the very different behavior between models with $E_{\text {bind, } \mathrm{H}_{2}}>465 \mathrm{~K}$ (indicated in blue) and those with $E_{\text {bind, } \mathrm{H}_{2}}<460 \mathrm{~K}$ (indicated in yellow).

respectively. This opens up a new type of chemistry leading to the species indicated in yellow, $\mathrm{CO}, \mathrm{HCO}, \mathrm{H}_{2} \mathrm{CO}, \mathrm{CO}_{2}$, and $\mathrm{HCOOH}$.
Methanol is not only a precursor for more complex saturated organic molecules (COMs) but also one of their main destruction products. We find that for high $\mathrm{H}_{2}$ binding energies, $\mathrm{CH}_{3} \mathrm{OH}$ is formed exclusively via hydrogenation of $\mathrm{CH}_{3} \mathrm{O}$ and $\mathrm{CH}_{2} \mathrm{OH}$, which are direct products of $\mathrm{H}_{2} \mathrm{CO}+\mathrm{H}$, even though most of the carbon is initially converted to $\mathrm{CH}_{4}$. For low $\mathrm{H}_{2}$ binding energies, however, the ice abundance of $\mathrm{HCO}$ increases, and $\mathrm{CH}_{3} \mathrm{O}$ and $\mathrm{CH}_{2} \mathrm{OH}$ are found to react with $\mathrm{HCO}$ instead of $\mathrm{H}$, leading to more complex species. In this way, methanol is more or less "skipped" in the reaction network and is predominantly formed through the destruction of more complex species (i.e., there opens up a "top-down" interstellar formation route to methanol ice).

For $E_{\text {bind, } \mathrm{H}_{2}}>465 \mathrm{~K}$, the $\mathrm{H}_{2}$ surface abundance was found to be equivalent to that of a thick $\mathrm{H}_{2}$ ice. This cannot realistically occur, since the binding energy of $\mathrm{H}_{2}$ to itself is significantly lower than that to the grain surface. For high $E_{\text {bind, } \mathrm{H}_{2}}$, a maximum of one monolayer of $\mathrm{H}_{2}$ is expected to cover the surface of the grain mantle. Heavier species landing on the grain will go through this layer to adsorb. $\mathrm{H}_{2}$ molecules landing or diffusing over another $\mathrm{H}_{2}$ molecule will desorb. We have changed our rate equation model to capture this behavior, roughly following Hincelin et al. (2015), as detailed in the Appendix. The correlation with $E_{\text {bind, } \mathrm{H}_{2}}$ is now found to disappear, as can be seen in Figure 3 with the lighter colors. In 
Table 6

Species with Pearson Correlation Coefficients $|P| \geqslant 0.3$ and Their Correspondent Values

\begin{tabular}{|c|c|c|c|c|c|c|}
\hline \multirow[b]{2}{*}{ Species } & \multicolumn{2}{|c|}{$1 \times 1 \times 10^{5}$ years } & \multicolumn{2}{|c|}{$5 \times 10^{5}$ years } & \multicolumn{2}{|c|}{$1 \times 10^{6}$ years } \\
\hline & corr. & $P$ & corr. & $P$ & corr. & $P$ \\
\hline \multirow[t]{2}{*}{$\overline{\mathrm{CH}_{3} \mathrm{OH}}$} & $\mathrm{HNO}$ & 0.39 & $\mathrm{CH}_{2}$ & 0.52 & $\mathrm{CH}_{2}$ & 0.50 \\
\hline & $\mathrm{CH}_{2}$ & 0.37 & & & $\mathrm{HCO}$ & 0.38 \\
\hline \multirow[t]{2}{*}{$\mathrm{CO}$} & $\mathrm{HCO}$ & 0.50 & $\mathrm{HCO}$ & 0.83 & $\mathrm{HCO}$ & 0.85 \\
\hline & $\mathrm{CH}_{2}$ & -0.33 & & & & \\
\hline \multirow[t]{3}{*}{$\mathrm{CO}_{2}$} & $\mathrm{CH}_{2}$ & -0.56 & $\mathrm{HCO}$ & -0.85 & $\mathrm{HCO}$ & -0.87 \\
\hline & $\mathrm{HNO}$ & 0.39 & & & & \\
\hline & $\mathrm{C}$ & -0.31 & & & & \\
\hline \multirow[t]{2}{*}{$\mathrm{H}_{2} \mathrm{O}$} & $\mathrm{HNO}$ & -0.66 & $\mathrm{HCO}$ & 0.55 & $\mathrm{HCO}$ & 0.65 \\
\hline & & & HNO & -0.52 & HNO & -0.41 \\
\hline \multirow[t]{3}{*}{$\mathrm{CH}_{4}$} & $\mathrm{C}$ & 0.66 & C & 0.62 & $\mathrm{C}$ & 0.57 \\
\hline & $\mathrm{HCO}$ & -0.33 & $\mathrm{HCO}$ & -0.38 & $\mathrm{HCO}$ & -0.43 \\
\hline & & & HNO & -0.30 & HNO & 0.3 \\
\hline \multirow[t]{2}{*}{$\mathrm{HCO}$} & $\mathrm{HCO}$ & 0.61 & $\mathrm{HCO}$ & 0.94 & $\mathrm{HCO}$ & 0.91 \\
\hline & $\mathrm{CH}_{2}$ & -0.33 & & & & \\
\hline \multirow[t]{2}{*}{$\mathrm{H}_{2} \mathrm{CO}$} & $\mathrm{HCO}$ & 0.78 & $\mathrm{HCO}$ & 0.93 & $\mathrm{HCO}$ & 0.8 \\
\hline & $\mathrm{C}$ & -0.36 & & & & \\
\hline \multicolumn{7}{|l|}{$\mathrm{NH}_{3}$} \\
\hline \multirow[t]{2}{*}{$\mathrm{HCOOH}$} & $\mathrm{HCO}$ & 0.55 & $\mathrm{HCO}$ & 0.66 & $\mathrm{HCO}$ & 0.32 \\
\hline & $\mathrm{C}$ & 0.31 & & & & \\
\hline
\end{tabular}

Note. Results are shown for $10^{5}, 5 \times 10^{5}$, and $10^{6}$ years.

the remainder of the paper, we will continue with these results, where the $\mathrm{H}_{2}$ surface abundance remains below one monolayer.

The dispersion found in Figure 3 is due to correlations with the binding energies of species other than $\mathrm{H}_{2}$. This is quantified again by the Pearson correlation function for a selection of ice species. All correlations with $|P| \geqslant 0.3$ are listed in Table 6 . As can be seen in this table, $\mathrm{CO}_{2}$ shows a strong correlation with the $\mathrm{HCO}$ binding energy at late times because an important formation route is through $\mathrm{HCO}+\mathrm{O}$. At early times, the correlation with $\mathrm{C}$ and $\mathrm{CH}_{2}$ can be explained by the formation of $\mathrm{CO}$ through $\mathrm{C}+\mathrm{O}$ and its competing reaction $\mathrm{C}+\mathrm{H}_{2}$ (see Figure 4). $\mathrm{HNO}$ is again linked to the formation of $\mathrm{OH}$, which is involved in $\mathrm{CO}_{2}$ production through $\mathrm{CO}+\mathrm{OH}$. Figure 3 also clearly shows the orders of magnitude difference between the ice abundances. To make the correlation between different abundances more apparent, we have performed a Principal Component Analysis (PCA) of the logarithm of the abundances of the main species obtained at $10^{6}$ years. In a PCA, an axis transformation is performed where the new dimensions, principal components, are linear combinations of the original dimensions, in this case the $\log$ of the ice abundances. These principal components are chosen such that the first describes as much of the variance in the data as is possible, followed by the second, etc. The results of the PCA analysis are shown in Figure 5. Each gray dot in this figure represents an individual simulation projected onto the first and second principal components (the scores). The spread of these points show that the dependence of the logarithm of the abundance on the parameter choice is highly nonlinear. The lines in the graph represent the loadings, which are the coefficients in the transformation matrix. The length of the line is a measure for the amount of variance in the original dimension. The length of the lines were multiplied by a factor of 10 for better visualization. In accordance with Figure 3, the species that present the largest variance in abundance are $\mathrm{CO}_{2}$ and $\mathrm{CH}_{4}$, followed by $\mathrm{H}_{2} \mathrm{CO}, \mathrm{HCO}, \mathrm{HCOOH}, \mathrm{CO}, \mathrm{HCOOH}, \mathrm{CH}_{3} \mathrm{OH}$,

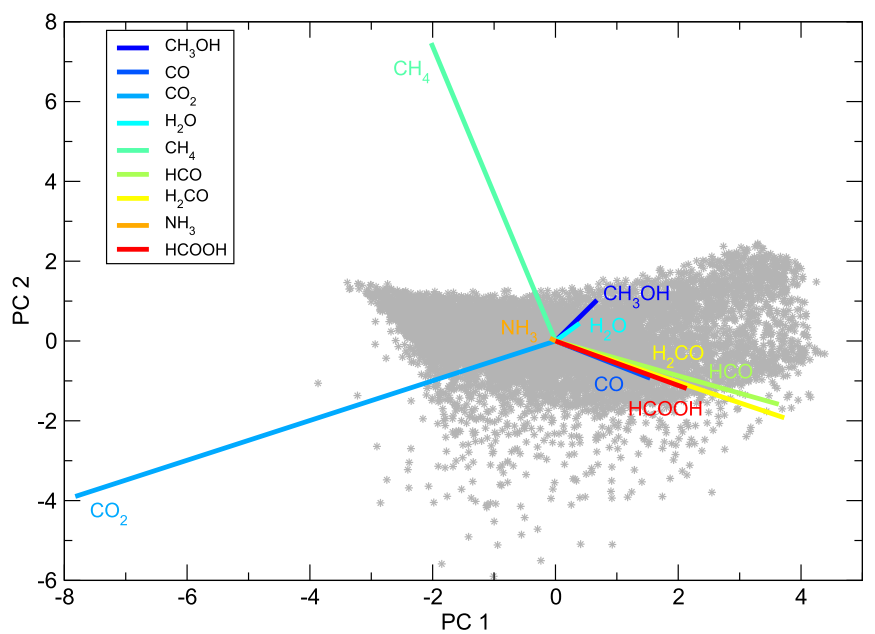

Figure 5. PCA of the sample of abundances for the main solid species obtained using low $\mathrm{H}_{2}$ binding energies. The distribution of abundances over the first and second principal components are shown in gray. The size of the bars represents the dispersion of abundances of each species, where the angle among the bars represents the strength of the correlation between the abundances of these species.

$\mathrm{H}_{2} \mathrm{O}$, and $\mathrm{NH}_{3}$, respectively. The directions of the lines reveal correlations between the abundances, where overlapping lines indicate species with similar variance patterns. Although it is dangerous to directly infer a chemical network from these correlates, we can however draw some conclusions, on the basis of the PCA in combination with the flux analysis that we performed earlier to arrive at Figure 4 . We see that the $\mathrm{CO}$ hydrogenation products, $\mathrm{HCO}, \mathrm{HCOOH}, \mathrm{H}_{2} \mathrm{CO}$, as well as $\mathrm{CO}$, are correlated. $\mathrm{CO}_{2}$ and $\mathrm{CH}_{3} \mathrm{OH}$, on the other hand, do not correlate as tightly, indicating that other routes are involved in their formation. $\mathrm{CO}_{2}$ nearly anticorrelates with $\mathrm{H}_{2} \mathrm{O}$, reflecting a similar precursor, $\mathrm{OH}$. $\mathrm{CH}_{4}$ and $\mathrm{NH}_{3}$ do not show a strong correlation with any of the other species, reflecting their rather separate formation routes. The PCA analysis hence confirms the scheme depicted in Figure 4.

\subsection{The HCO Radical and the Importance of Branching Ratios}

The Pearson correlation coefficients and the network depicted in Figure 4 show that HCO plays a pivotal role in the grain-surface network. Here we will look at its formation and destruction routes more closely. Its main formation routes are through hydrogenation of $\mathrm{CO}$ and $\mathrm{H}_{2} \mathrm{CO}$; destruction also mainly occurs through hydrogenation of $\mathrm{H}_{2} \mathrm{CO}$. The reaction $\mathrm{H}+\mathrm{H}_{2} \mathrm{CO}$ has three product channels: formation of $\mathrm{CH}_{3} \mathrm{O}$ or $\mathrm{CH}_{2} \mathrm{OH}$ and abstraction of a hydrogen atom leading to $\mathrm{HCO}$ and $\mathrm{H}_{2}$. Using the standard network, the combination of reaction rates and branching ratios leads to an effective branching ratio of 0.97 for the latter channel. The remainder is equally shared between $\mathrm{CH}_{3} \mathrm{O}$ or $\mathrm{CH}_{2} \mathrm{OH}$. Whereas this might reflect the gas-phase chemistry, we find, however, no laboratory data to support these extreme branching ratios on the surface. In a recent experimental study, Chuang et al. (2016) investigated these reactions, but they do not quote branching ratios. They find that, indeed, the addition reaction to $\mathrm{CH}_{3} \mathrm{O}$ and the abstraction to $\mathrm{H}_{2}+\mathrm{HCO}$ occur, and they cannot exclude the channel to form $\mathrm{CH}_{2} \mathrm{OH}$. Here, we alter the network by adopting equal ratios for all three channels and performed 100 additional simulations using this network. The evolution of the ice abundance of the species involved in the 


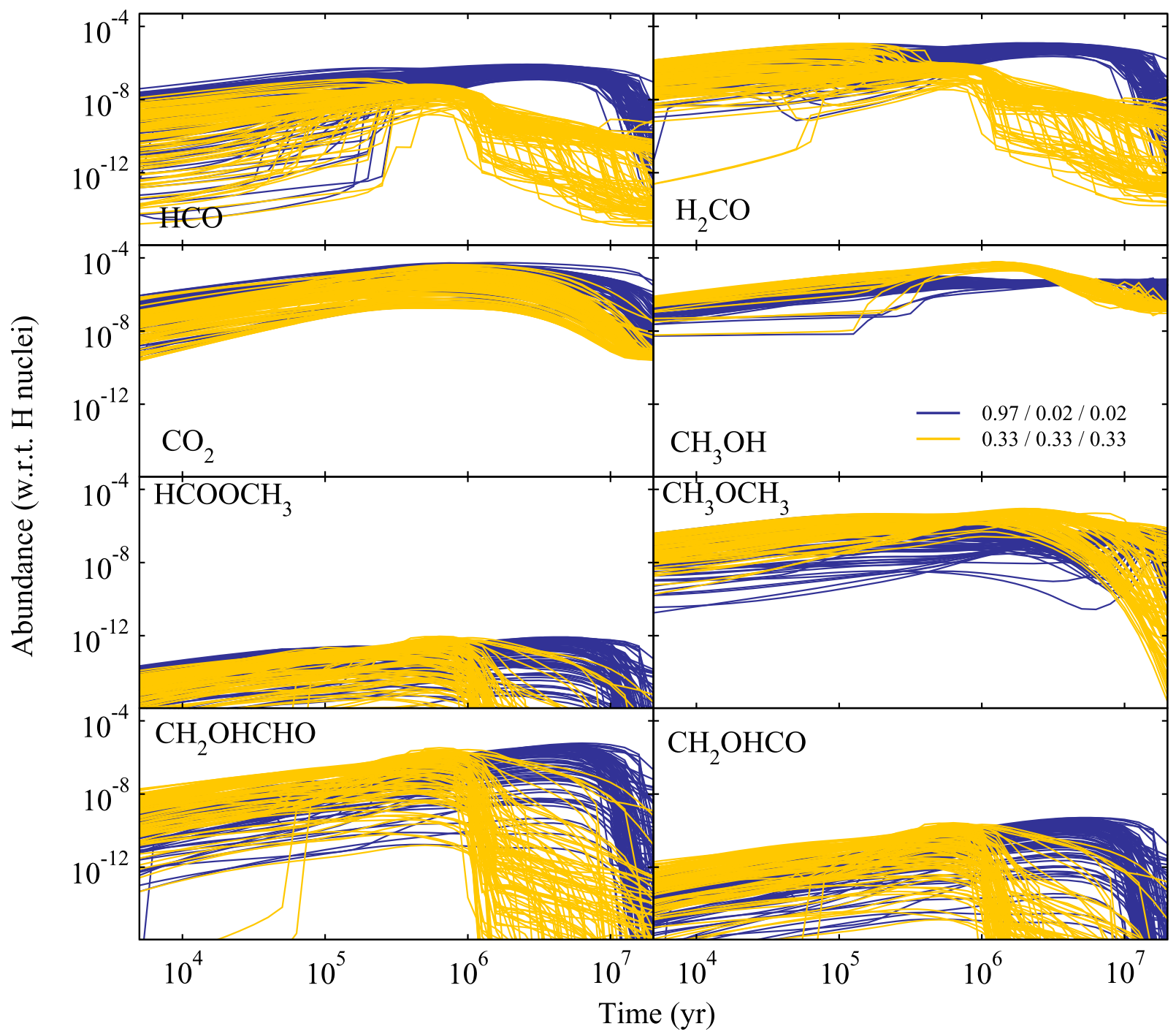

Figure 6. Evolution of ice abundances according to two different branching ratios: 0.97/0.02/0.02 (standard network) and 0.33/0.33/0.33 (updated network). Results using the standard network are shown in blue and using the updated network in yellow. Abundances much lower than $10^{-16}$ are negligible and are therefore not shown here.

hydrogenation of $\mathrm{CO}$ is shown in Figure 6 in yellow. A comparison is made between the original 100 runs of our standard network in blue.

The timescale of conversion of $\mathrm{HCO}$ to $\mathrm{H}_{2} \mathrm{CO}$ and $\mathrm{CH}_{3} \mathrm{OH}$ is reduced by a few million years, since there are less backreactions. Moreover, as a consequence of the reduction in $\mathrm{HCO}$, an increase in the methanol abundance can be observed, since $\mathrm{CH}_{3} \mathrm{O}$ and $\mathrm{CH}_{2} \mathrm{OH}$ will now predominantly react with $\mathrm{H}$ to form methanol. For the more complex species, like $\mathrm{HCOOCH}_{3}, \mathrm{CH}_{2} \mathrm{OHCHO}$, and the intermediate radical $\mathrm{CH}_{2} \mathrm{OHCO}$, both a reduction in the peak intensity and a change in the formation timescale can be observed.

This example clearly shows the importance of accurate branching ratios. In this case, for grain-surface reactions with multiple product channels, the ratio between the forwards and backwards reactions is crucial since this determines the timescale of $\mathrm{CH}_{3} \mathrm{OH}$ formation and the route of complex molecule formation: through $\mathrm{CH}_{3} \mathrm{OH}$ or through reactions with HCO. We conclude that, using the standard network, reactions forming complex molecules involving $\mathrm{HCO}$ are likely overexpressed. Numerous other reactions in the grain-surface network have several reaction channels, and we believe that the branching ratios used should be carefully scrutinized as it is possible that they have strong effects.

\subsection{The Effect of Initial Conditions on COM Production}

The overall results indicate that the $\mathrm{C}+\mathrm{O} \rightarrow \mathrm{CO}$ reaction is crucial in the formation of COMs through competing surface routes as depicted in Figure 4. So far, the initial form of elemental carbon in the simulations is chosen to be atomic carbon. However, depending on the history of the molecular cloud, a substantial fraction could also already be in the form of CO. In this case, one would expect the methanol route to start earlier. Here we will look at the extreme case in which all elemental carbon is initially in the form of CO.

We follow a similar procedure to the previous section by performing another 100 simulations, but with all carbon initially in the form of $\mathrm{CO}$. The initial atomic oxygen abundance is reduced accordingly. The standard network is used and the results are again compared to the previous results. The results are shown in Figure 7. These show an unexpected reduction in $\mathrm{H}_{2} \mathrm{CO}, \mathrm{CH}_{3} \mathrm{OH}$, and $\mathrm{COMs}$ abundance when we start with all $\mathrm{C}$ in the form of $\mathrm{CO}$. The crucial radical to explain 


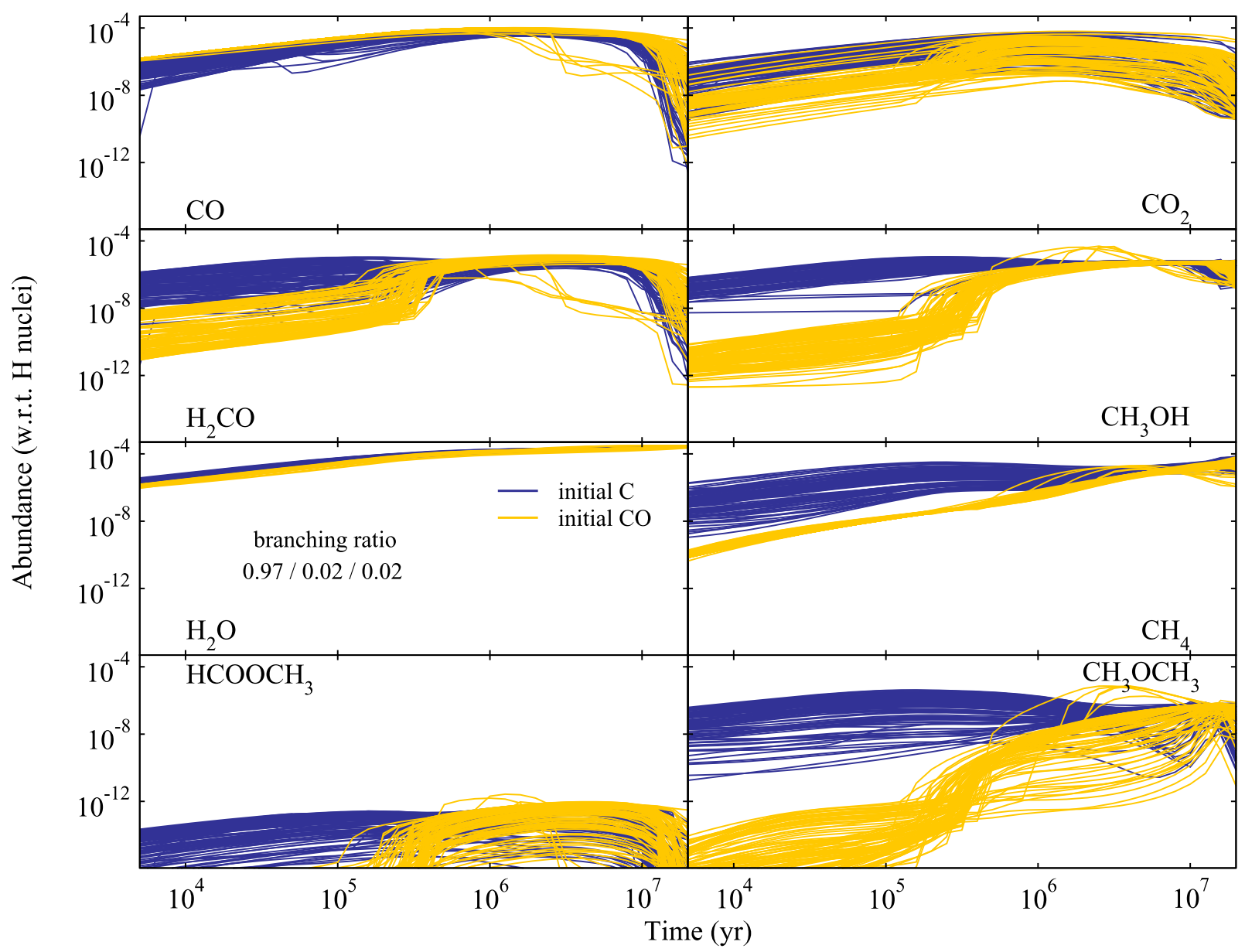

Figure 7. Comparison between the evolution of selected ice-phase species using different initial conditions. Dark colors show simulations using the elemental form of carbon as initial conditions, while light colors refer to simulations using $\mathrm{CO}$ as the initial form of carbon. Each panel shows 100 simulations for each initial condition, where 50 runs use $E_{\text {bind, } \mathrm{H}_{2}}>465 \mathrm{~K}$ (dark and light blue) and the other 50 runs using $E_{\text {bind, } \mathrm{H}_{2}}<460 \mathrm{~K}$ (orange and yellow). Here, the standard network is used, i.e., the branching ratio of $0.97 / 0.02 / 0.02$ for the hydrogenation reactions of $\mathrm{H}_{2} \mathrm{CO}$. Abundances much lower than $10^{-16}$ are negligible and are therefore not shown here.

this effect is $\mathrm{CH}_{3}$. By starting exclusively from $\mathrm{CO}$, the production rate of this radical is reduced by at least an order of magnitude. It can react with, for instance, $\mathrm{HCO}$ to form $\mathrm{CH}_{3} \mathrm{CHO}$, which can fall back to $\mathrm{H}_{2} \mathrm{CO}$ upon hydrogenation or lead to more complex molecules, which in turn reduce to methanol again. Overall, this careful analysis of the reaction network shows that rather than being a final product, COMs also play a crucial role as an intermediate to form main grain mantle species such as formaldehyde and methanol. This already occurs in dark conditions and no photoprocesses are needed for COMs to form at low temperatures ( $10 \mathrm{~K})$. In hot core models, the photodissociation of methanol is used as the formation mechanism of radical species that can then recombine to form more complex molecules upon warm up triggered by the birth of the central star (Garrod \& Herbst 2006; Garrod et al. 2008). Here, we see that they may already form at low temperatures, but that they are destroyed by abstraction reactions with atomic hydrogen. The latter reactions become less important for higher temperatures since the residence time of $\mathrm{H}$ atoms is significantly reduced at temperatures above $20 \mathrm{~K}$.

\subsection{Comparison with Ice Observations}

Distributions of the obtained ice abundances with respect to $\mathrm{H}$ nuclei are shown in Figure 8 for $\mathrm{H}_{2} \mathrm{O}, \mathrm{CO}, \mathrm{CO}_{2}, \mathrm{CH}_{3} \mathrm{OH}$, $\mathrm{NH}_{3}$, and $\mathrm{CH}_{4}$, which are all securely identified in these type of objects. Despite the large apparent dispersion in the ice abundances in Figure 3, these histograms highlight that the dispersion in terms of the FWHM is significantly narrower $(\lesssim 1$ order of magnitude) for most species. We compare the obtained model abundances with observational abundances taken from a recent review by Boogert et al. (2015) where we have taken the background star observations as representative of observations of quiescent clouds and cores. What is clear is the very large spread in observational abundances as indicated by the yellow areas in Figure 8, from upper limits to relatively large values compared with water ice observations. Inspection of Figure 8 shows that the best agreement is obtained at relatively early times of $10^{5}$ years. This coincides with dark cloud model results focusing on the gas phase, where $10^{5}$ years is considered as the early time of best overall agreement between models and observations for a large number of species. The current understanding is that the relatively low density of $n_{\mathrm{H}}=2 \times 10^{4} \mathrm{~cm}^{-3}$ persists until roughly $1 \mathrm{Myr}$, when the density increases and the remaining gas-phase $\mathrm{CO}$ freezes out rapidly on the grains and the star formation sequence starts. With the current $n_{\mathrm{H}}$ density, CO freeze out occurs roughly in $2 \times 10^{5}$ years (see Figure 7). After this point, the CO adsorption from the gas phase reduces and the destruction of the more complex species wins over formation, simply because its main precursor has run out. 


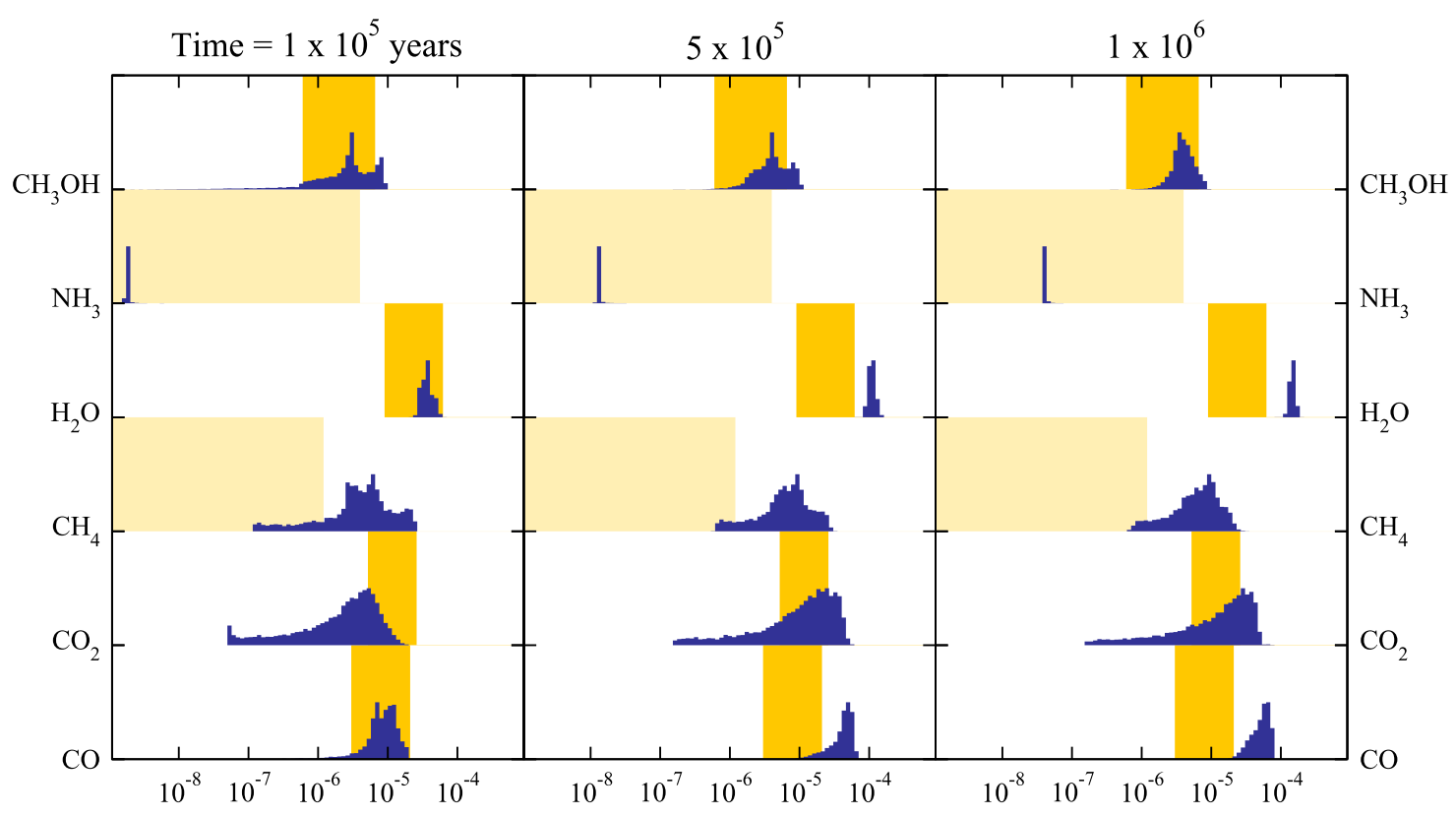

Abundance (w.r.t. H nuclei)

Figure 8. Distributions of simulated ice abundances (blue histograms) for three different times ( $10^{5}$ years in the left, $5 \times 10^{5}$ years in the middle, and $10^{6}$ years in the right panels) compared to abundances derived from observations (yellow areas, light yellow for upper limits) of quiescent clouds and cores (see Boogert et al. 2015 for references).

The observations of $\mathrm{CH}_{4}$ and $\mathrm{NH}_{3}$ show only upper limits. Our model results for $\mathrm{NH}_{3}$ fall well below this limit, whereas a large fraction of models heavily overproduce $\mathrm{CH}_{4}$. This overproduction of $\mathrm{CH}_{4}$ could be due to too efficient destruction reactions of COMs, because of inaccuracy in branching ratios of reactions leading to $\mathrm{CH}_{4}$ or its precursors, justifying a complete scrutinization of the whole network.

\section{Discussion and Conclusions}

With this paper, we set out to study the influence of binding energies of surface species on simulated ice abundances in order to constrain some of the binding energies. We found that studying the correlations between binding energies and simulated ice abundances served as an excellent tool to scrutinize the reaction network. As a result, we obtained more information on the reaction network than actually constraining certain binding energies. One reason for this could be the low temperature of $10 \mathrm{~K}$ applied in the simulations. At these temperatures, most species are stationary for their full range of binding energies. Hot core or disk models that probe a larger spread in temperature might be more suited for this purpose. However, analyzing the data of a simple dark cloud to really understand the dependencies is already very complex and hence serves as a good starting point for similar studies of more complex astrophysical environments, and we leave this to future work. Finally, we want to stress that only the binding energies were varied and that all other parameters are held constant. We expect these to have an effect as well, in particular the diffusionto-desorption ratio. This is also a topic for future study. Moreover, it would be valuable to extend the present work to a more realistic three-phase model, so that the bulk and the grain surface can be treated separately. Such a model has been shown to capture some of the complexity that arises for the desorption of mixtures of light species such as $\mathrm{CO}$, which exhibits multiple desorption peaks (Fayolle et al. 2011). We arrived at the following set of conclusions and recommendations.

1. For a high binding energy of $\mathrm{H}_{2}$, rate equations can result in the unrealistic build up of $\mathrm{H}_{2}$ ice. Rate equations should be corrected for this, since a high surface abundance of $\mathrm{H}_{2}$ can trigger a different type of surface chemistry.

2. For dark cloud models, the binding energies of $\mathrm{C}, \mathrm{HCO}$, $\mathrm{HNO}$, and $\mathrm{CH}_{2}$ are the most determining for the final ice abundances. Since diffusion barriers are inferred from binding energies, these dependencies most likely involve diffusion rates. Obtaining accurate diffusion rates or binding energies for these species would result in a significant improvement in the reliability of grain-surface models.

3. $\mathrm{HNO}$ is actively involved in the production of $\mathrm{OH}$. Hydrogenation reactions of HNO should be included in the network, in accordance with laboratory results, to prevent the overproduction of $\mathrm{OH}$.

4. The branching ratios of reaction products resulting from the hydrogenation of $\mathrm{H}_{2} \mathrm{CO}$ were found to play a crucial role in determining the timescale for $\mathrm{COM}$ formation as well as their formation routes. We believe that with the network used here, reactions forming complex molecules involving $\mathrm{HCO}$ are too efficient. We encourage experimentalists to work toward extracting branching ratio information from laboratory data: this work highlights the importance of accurate branching ratios for the outcome of grain-surface models. Since numerous other reactions in the grain-surface network have several reaction channels, we recommend modelers to carefully scrutinize the branching ratios they use as these ratios can have strong effects.

5. COMs play a crucial role as intermediates for formaldehyde and methanol and are not only the final products in 
the reaction network. COMs are thus mainly formed through reactions with $\mathrm{HCO}$, the abundance of which highly depends on the $\mathrm{H}+\mathrm{H}_{2} \mathrm{CO}$ branching ratio, and subsequently destroyed by abstraction reactions with atomic $\mathrm{H}$.

E.M.P. and H.M.C. acknowledge the European Research Council (ERC-2010-StG, Grant Agreement no. 259510-KISMOL) for financial support. H.M.C. is grateful for support from the VIDI research program 700.10.427, which is financed by The Netherlands Organization for Scientific Research (NWO). C.W. acknowledges support from the VENI research program 639.041.335, also financed by NWO, and start-up funds from the University of Leeds. We would like to thank Jeroen Jansen (Radboud University) for fruitful discussions about the application of PCA and Alexander Atamas for computational support.

\section{Appendix}

Rate equations do not have any positional information of the species. Species in the top layer are, in principle, treated in the same way as in the bulk of the ice. This results in too many species that actively participate in surface reactions and in the wrong desorption order. The current rate equation models applies a fix to artificially account for this, as explained in Cuppen et al. (2017). In this treatment, surface reactions do not depend on the number of reactants on the grain $\left(n_{\mathrm{S}}(A) n_{\mathrm{s}}(B)\right.$ for the reaction $A+B \rightarrow C$ ) but on the number of reactants in the active layer of size $N_{\text {act }}\left(\chi(A) N_{\text {act }} \chi(B) N_{\text {act }}\right.$ with $\left.\chi(A)=n_{\mathrm{s}}(A) / \sum_{X} n_{\mathrm{s}}(X)\right)$. Hence, a homogeneous distribution of all species is assumed throughout the grain mantle. If the total number of species on the grain $\sum_{X} n_{\mathrm{s}}(X)$ is less than $N_{\text {act }}$, the original expression with is $n_{\mathrm{s}}(A) n_{\mathrm{s}}(B)$ is used.

Molecular hydrogen is assumed to reside exclusively on top of the grain mantle. It should hence be limited to $N_{\text {act }}$. We follow the mechanism of Hincelin et al. (2015) to account for the desorption of any additional $\mathrm{H}_{2} \cdot \mathrm{H}_{2}$ molecules that diffuse on top of a $\mathrm{H}_{2}$ molecule in a layer below is assumed to desorb. Because of the fix we apply for surface reactions, the additional term in the rate equation deviates from the original expression in Hincelin et al. (2015):

$$
R_{\mathrm{H}_{2} \text { desorb }}=\exp \left(-\frac{E_{\mathrm{bind}, \mathrm{H}_{2} \text { to }, \mathrm{H}_{2}}}{k_{\mathrm{B}} T}\right) k_{\text {diff }} \frac{N_{\mathrm{act}}^{2}}{N_{\text {grain }}} \theta\left(\frac{n_{\mathrm{s}}\left(\mathrm{H}_{2}\right)}{N_{\mathrm{act}}}-\theta\right),
$$

where

$$
\theta= \begin{cases}\frac{n_{\mathrm{s}}\left(\mathrm{H}_{2}\right)}{N_{\mathrm{act}}}, & \text { if } N_{\mathrm{H}}<N_{\mathrm{act}} \\ \frac{n_{\mathrm{s}}\left(\mathrm{H}_{2}\right)}{N_{\mathrm{H}}}, & \text { otherwise }\end{cases}
$$

and $N_{\mathrm{H}}=n_{\mathrm{s}}(\mathrm{H})+n_{\mathrm{s}}\left(\mathrm{H}_{2}\right)$. Choosing $E_{\text {bind, } \mathrm{H}_{2}}$ to $\mathrm{H}_{2}$ results in a maximum coverage of $N_{\text {act }}$ for $\mathrm{H}_{2}$.

\section{References}

Acharyya, K. 2014, MNRAS, 443, 1301

Acharyya, K., Fuchs, G. W., Fraser, H. J., van Dishoeck, E. F., \& Linnartz, H. 2007, A\&A, 466, 1005

Aikawa, Y., Miyama, S. M., Nakano, T., \& Umebayashi, T. 1996, ApJ, 467, 684

Al-Halabi, A., \& van Dishoeck, E. F. 2007, MNRAS, 382, 1648

Allen, M., \& Robinson, G. W. 1977, ApJ, 212, 396

Boogert, A. A., Gerakines, P. A., \& Whittet, D. C. 2015, ARA\&A, 53, 541

Brown, W. A., \& Bolina, A. S. 2007, MNRAS, 374, 1006

Charnley, S. B. 1997, MNRAS, 291, 455

Chuang, K. J., Fedoseev, G., Ioppolo, S., van Dishoeck, E. F., \& Linnartz, H. 2016, MNRAS, 455, 1702

Collings, M. P., Anderson, M. A., Chen, R., et al. 2004, MNRAS, 354 1133

Collings, M. P., Dever, J. W., Fraser, H. J., \& McCoustra, M. R. S. 2003, Ap\&SS, 285, 633

Collings, M. P., Frankland, V. L., Lasne, J., et al. 2015, MNRAS, 449, 1826

Congiu, E., Fedoseev, G., Ioppolo, S., et al. 2012, ApJL, 750, L12

Cuppen, H. M., van Dishoeck, E. F., Herbst, E., \& Tielens, A. G. G. M. 2009, A\&A, 508, 275

Cuppen, H. M., Walsh, C., Lamberts, T., et al. 2017, SSRv, in press

Drozdovskaya, M. N., Walsh, C., Visser, R., Harsono, D., \& van Dishoeck, E. F. 2014, MNRAS, 445, 913

Drozdovskaya, M. N., Walsh, C., Visser, R., Harsono, D., \& van Dishoeck, E. F. 2015, MNRAS, 451, 3836

Dulieu, F., Congiu, E., Noble, J., et al. 2013, NatSR, 3, 1338

Fayolle, E. C., Öberg, K. I., Cuppen, H. M., Visser, R., \& Linnartz, H. 2011, A\&A, 529, A74

Fedoseev, G., Ioppolo, S., Lamberts, T., et al. 2012, JChPh, 137, 054714

Fraser, H. J., Collings, M. P., McCoustra, M. R. S., \& Williams, D. A. 2001, MNRAS, 327, 1165

Fuchs, G. W., Acharyya, K., Bisschop, S. E., et al. 2006, FaDi, 133, 331

Garrod, R. T., \& Herbst, E. 2006, A\&A, 457, 927

Garrod, R. T., Vasyunin, A. I., Semenov, D. A., Wiebe, D. S., \& Henning, T. 2009, ApJL, 700, L43

Garrod, R. T., Weaver, S. L. W., \& Herbst, E. 2008, ApJ, 682, 283

Graedel, T. E., Langer, W. D., \& Frerking, M. A. 1982, ApJS, 48, 321

Green, S. D., Bolina, A. S., Chen, R., et al. 2009, MNRAS, 398, 357

Hasegawa, T. I., \& Herbst, E. 1993a, MNRAS, 261, 83

Hasegawa, T. I., \& Herbst, E. 1993b, MNRAS, 263, 589

Hasegawa, T. I., Herbst, E., \& Leung, C. M. 1992, ApJS, 82, 167

He, J., Jing, D., \& Vidali, G. 2014, PCCP, 16, 3493

He, J., \& Vidali, G. 2014, ApJ, 788, 50

Hincelin, U., Chang, Q., \& Herbst, E. 2015, A\&A, 574, A24

Indriolo, N., Neufeld, D. A., Gerin, M., et al. 2015, ApJ, 800, 40

Karssemeijer, L. J., \& Cuppen, H. M. 2014, A\&A, 569, A107

McElroy, D., Walsh, C., Markwick, A. J., et al. 2013, A\&A, 550, A36

Noble, J. A., Congiu, E., Dulieu, F., \& Fraser, H. J. 2012a, MNRAS, 421, 768

Noble, J. A., Theulé, P., Mispelaer, F., et al. 2012b, A\&A, 543, A5

Öberg, K. I., van Broekhuizen, F., Fraser, H. J., et al. 2005, ApJL, 621, L33

Ruffle, D. P., \& Herbst, E. 2000, MNRAS, 319, 837

Sandford, S. A., \& Allamandola, L. J. 1988, Icar, 76, 201

Sandford, S. A., Allamandola, L. J., Tielens, A. G. G. M., \& Valero, G. J. 1988, ApJ, 329, 498

Smith, R. S., May, R. A., \& Kay, B. D. 2016, JPCB, 120, 1979

Tielens, A. G. G. M., \& Allamandola, L. J. 1987, in Interstellar Processes, ed. D. J. Hollenbach \& H. A. Thronson, Jr. (Dordrecht: Reidel), 397

Tielens, A. G. G. M., \& Hagen, W. 1982, A\&A, 114, 245

Wakelam, V., Herbst, E., Le Bourlot, J., et al. 2010, A\&A, 517, A21

Wakelam, V., Herbst, E., Selsis, F., \& Massacrier, G. 2006, A\&A, 459, 813

Walsh, C., Nomura, H., \& van Dishoeck, E. 2015, A\&A, 582, A88 